\title{
Geometric Models for Quasicrystals I. Delone Sets of Finite Type
}

\author{
J. C. Lagarias
}

AT\&T Labs, 180 Park Avenue, Florham Park, NJ 07932, USA

jcl@research.att.com

\begin{abstract}
This paper studies three classes of discrete sets $X$ in $\mathbb{R}^{n}$ which have a weak translational order imposed by increasingly strong restrictions on their sets of interpoint vectors $X-X$. A finitely generated Delone set is one such that the abelian group $[X-X]$ generated by $X-X$ is finitely generated, so that $[X-X]$ is a lattice or a quasilattice. For such sets the abelian group $[X]$ is finitely generated, and by choosing a basis of $[X]$ one obtains a homomorphism $\varphi:[X] \rightarrow \mathbb{Z}^{s}$. A Delone set of finite type is a Delone set $X$ such that $X-X$ is a discrete closed set. A Meyer set is a Delone set $X$ such that $X-X$ is a Delone set.

Delone sets of finite type form a natural class for modeling quasicrystalline structures, because the property of being a Delone set of finite type is determined by "local rules." That is, a Delone set $X$ is of finite type if and only if it has a finite number of neighborhoods of radius $2 R$, up to translation, where $R$ is the relative denseness constant of $X$. Delone sets of finite type are also characterized as those finitely generated Delone sets such that the map $\varphi$ satisfies the Lipschitz-type condition $\left\|\varphi(\mathbf{x})-\varphi\left(\mathbf{x}^{\prime}\right)\right\|<C\left\|\mathbf{x}-\mathbf{x}^{\prime}\right\|$ for $\mathbf{x}, \mathbf{x}^{\prime} \in X$, where the norms $\|\cdot\|$ are Euclidean norms on $\mathbb{R}^{s}$ and $\mathbb{R}^{n}$, respectively. Meyer sets are characterized as the subclass of Delone sets of finite type for which there is a linear map $\tilde{L}: \mathbb{R}^{n} \rightarrow \mathbb{R}^{s}$ and a constant $C$ such that $\|\varphi(\mathbf{x})-\tilde{L}(\mathbf{x})\| \leq C$ for all $\mathbf{x} \in X$.

Suppose that $X$ is a Delone set with an inflation symmetry, which is a real number $\eta>1$ such that $\eta X \subseteq X$. If $X$ is a finitely generated Delone set, then $\eta$ must be an algebraic integer; if $X$ is a Delone set of finite type, then in addition all algebraic conjugates $\left|\eta^{\prime}\right| \leq \eta$; and if $X$ is a Meyer set, then all algebraic conjugates $\left|\eta^{\prime}\right| \leq 1$.
\end{abstract}

\section{Introduction}

The discovery of quasicrystalline materials in 1984 has generated much theoretical and experimental work [10], [29], [30], [56], [77]. These are materials which have X-ray 
diffraction spectra that have sharp spots ("Bragg peaks") indicating long range order of their atomic structure under translations, but which exhibit forbidden symmetries indicating that they cannot have crystallographic atomic structure. The list of quasicrystalline materials now includes a few types of thermodynamically stable "perfect quasicrystals" which have structure as uniform as crystals, as well as a much larger variety of quasicrystalline structures with less perfect order [20]. There are a number of different structural models proposed for quasicrystalline materials, ranging from random tiling models [22], [23] to models based on cut-and-project sets [37], [43], [44]. Indeed such structures can exhibit diffraction patterns of approximately the right kind [12], [24], [29], [53]. This series of papers is motivated by the problem of finding atomic models for such materials, that can describe both thermodynamically stable quasicrystals and more disordered quasicrystalline structures. In particular, we consider sets with weaker kinds of long-range order under translation that would not necessarily be manifested by "Bragg peaks" in their diffraction spectrum.

The concept of a Delone set is a mathematical abstraction for the positions of atoms in a solid state material.

Definition 1.1. A discrete set $X$ is a Delone set (or Delaunay set or $(r, R)$-set) if it satisfies the following two conditions:

(i) Uniform Discreteness. There is a value $r$ such that each open ball of radius $r$ contains at most one point of $X$.

(ii) Relative Denseness. There is a value $R$ such that each closed ball of radius $R$ contains at least one point of $X$.

The maximal value of $r$ is the packing radius for $X$ using equal spheres centered at its points, and the minimal value of $R$ is the covering radius of $X$ using equal spheres centered at its points to cover $\mathbb{R}^{n}$.

The notion of Delone sets as the fundamental object of study in crystallography was proposed by the Russian school in the 1930s, see [8], [9], [19], and p. 25 of [71]. Delone set models for quasicrystals have been studied by a number of authors, e.g., Danzer [5, p. 571] and Klitzing and Baake [35].

In this paper we consider three classes of discrete geometric structures in $\mathbb{R}^{n}$, which consist of Delone sets $X$ with some translational order imposed by restrictions on their set of interpoint vectors $X-X$. It is natural to consider restrictions on the set of interpoint vectors $X-X$, first because the diffraction pattern is determined by the set $X-X$, and second because restrictions on local arrangements of atoms appear to be imposed in some materials by local energy minimization. ${ }^{1}$

The three classes of Delone sets that we study are specified by increasingly strong restrictions on their sets of interpoint vectors $X-X$, as follows.

\footnotetext{
${ }^{1}$ The resultant of the forces acting on each atom must be zero (local stability). The electromagnetic force is long range, but the dominant effects on one atom come from nearby atoms due to approximate cancellation of forces from atoms further away.
} 
Definition 1.2. Let $X$ be a Delone set.

(i) $X$ is a finitely generated Delone set if the abelian group

$$
[X-X]=\mathbb{Z}\left[\mathbf{x}-\mathbf{x}^{\prime}: \text { all } \mathbf{x}, \mathbf{x}^{\prime} \in X\right]
$$

is finitely generated.

(ii) $X$ is a Delone set of finite type if $X-X$ is a discrete closed subset of $\mathbb{R}^{n}$, i.e., the intersection of $X-X$ with any closed ball is a finite set.

(iii) $X$ is a Meyer set if $X-X$ is a Delone subset of $\mathbb{R}^{n}$.

We show that class (i) includes class (ii), and class (ii) obviously includes class (iii). The terminology "Delone set of finite type" is motivated by the fact that Delone sets of finite type have only finitely many types of local neighborhoods of radius $2 R$ under translations, see Section 2. More precisely (ii) defines Delone sets of finite type under translations, to distinguish them from the larger class of Delone sets of finite type under isometries considered in [11] and [39]. The class of Meyer sets was introduced by Meyer [53] under the name "quasicrystal"; they are now generally called Meyer sets, see [38], [54], and [55]. Definition (iii) of a Meyer set is not the usual one, but in [38] it is shown equivalent to Meyer's original definition that $X-X \subseteq X+F$, for some finite set $F$. Meyer [53] observes that all cut-and-project sets are Meyer sets, and presents evidence that they form a suitable class to be termed quasicrystals. We argue here that the more inclusive class of Delone sets of finite type is the natural general class of sets to consider in making models for the atomic structure of quasicrystalline materials. However, both of these classes include sets that are not quasicrystalline in the sense of possessing a well-defined diffraction spectrum. Various examples that illustrate these points appear in Section 5.

A Delone set $X$ is finitely generated if and only if the abelian group

$$
[X]:=\mathbb{Z}[\mathbf{x}: \mathbf{x} \in X]
$$

is finitely generated. We view this property as a weak form of long-range order under translations ${ }^{2}$ of the elements of $X$. A finitely generated abelian group $[X]$ that spans $\mathbb{R}^{n}$ is called a quasilattice; it is called a lattice if it is discretely embedded in $\mathbb{R}^{n}$. Quasilattices have been introduced as a unifying concept in studying the symmetries of quasicrystals, see [42], [50], and [60]. The rank of $X$ is the number of generators of $[X]$ as a free abelian group. (All finitely generated subgroups of $\mathbb{R}^{n}$ are free.)

Definition 1.3. Let $X$ be a finitely generated Delone set with $\operatorname{rank}(X)=s$, and choose a basis of $[X]$, say

$$
[X]=\mathbb{Z}\left[\mathbf{v}_{1}, \mathbf{v}_{2}, \ldots, \mathbf{v}_{s}\right] .
$$

The address map $\varphi:[X] \rightarrow \mathbb{Z}^{s}$ associated to this basis is

$$
\varphi\left(\sum_{i=1}^{s} n_{i} \mathbf{v}_{i}\right)=\left(n_{1}, n_{2}, \ldots, n_{s}\right) .
$$

\footnotetext{
${ }^{2}$ See the note on terminology at the end of the introduction.
} 
The address map is specified by the choice of basis of $[X]$, so it is determined up to left-multiplication by an element of $G L(s, \mathbb{Z})$. The structure of a finitely generated Delone set is to some extent analyzable by studying its image in $\mathbb{R}^{s}$ under the address map. Using the address map the points of $X$ can be coordinatized as a subset $Y$ of the integer lattice $\mathbb{Z}^{s}$ regarded as embedded in $\mathbb{R}^{s}$, with $s=\operatorname{rank}(X)$, and there is a linear projection $\psi: \mathbb{R}^{s} \rightarrow \mathbb{R}^{n}$ with $\psi(Y)=X$. The address map describes $X$ using $s$ "internal dimensions." In this coordinatization all $s$ dimensions are on an equal footing; there seems no natural way to select $n$ of these dimensions as "physical dimensions."

The class of finitely generated Delone sets is the largest class of sets on which an address map is well defined. It seems to be too large a class to be interesting, but membership in it does put algebraic restrictions on inflation symmetries, as described in Section 4. Various interesting subclasses of such sets are obtained by putting restrictions on the address map which require that points that are close in $X$ have addresses which are not too far apart in the address space $\mathbb{R}^{s}$. The most important subclass of such sets seems to be Delone sets of finite type, which we show are characterized by a Lipschitz-type condition on the address map.

The class of Delone sets of finite type appears to be a natural unifying concept in classifying quasicrystalline structures. In Section 2 we show that Delone sets of finite type form the largest class of Delone sets that can be described by "local rules under translations." That is, we show that a Delone set $X$ is of finite type if and only if $X$ has only a finite number of different local neighborhoods of radius $2 R$ around its points $\mathbf{x} \in X$, up to translation (Theorem 2.1). This result shows that the property of being a Delone set of finite type is enforceable by local rules under translations, in the sense of part II, see also [42] and [45]. It also follows that a Delone set $X$ of finite type in $\mathbb{R}^{n}$ is always finitely generated, and $\operatorname{rank}(X)$ is bounded by the number of points in $X-X$ of length at most $2 R$.

One way to model the atomic structure of quasicrystalline materials which have X-ray diffraction patterns which exhibit crystallographically forbidden symmetries is through the use of sets described using a finite number of extra "internal dimensions," see [50]. Certain cut-and-project sets provide examples; they are obtained as projections of part of a higher-dimensional lattice, see Section 3. To get a Delone set in $\mathbb{R}^{3}$ having a diffraction pattern having "Bragg peaks" located at points with icosahedral symmetry, one can project from a suitable six-dimensional lattice. The "local rules under translations" result above for Delone sets of finite type shows that finiteness of the number of "internal dimensions" describing a set follows as a consequence of finiteness of the number of allowable local configurations under translations. In part II we strengthen this result by showing that finiteness of the number of "internal dimensions" can also be forced by local rules under isometries, for a large class of structures.

Tiling models of quasicrystals naturally produce Delone sets of finite type. Consider a tiling of $\mathbb{R}^{n}$ which consists of translates of finitely many types of prototiles. We associate a Delone set $X$ to the tiling by marking a finite set of points in each prototile ("decorations"), see, for example, [35]. If there are only a finite number of different ways two prototiles may be adjacent in this tiling, then $X$ is necessarily a Delone set of finite type, and conversely (Corollary 2.1). In particular, random tiling models of quasicrystals in the sense of Henley [23, Section 2.3] yield Delone sets of finite type.

In Section 2 we give several characterizations of Delone sets of finite type. We show 
that they are characterized by either of the following conditions:

(1) The address map $\varphi$ satisfies the Lipschitz-type condition

$$
\left\|\varphi(\mathbf{x})-\varphi\left(\mathbf{x}^{\prime}\right)\right\| \leq C\left\|\mathbf{x}-\mathbf{x}^{\prime}\right\| \quad \text { for } \quad \mathbf{x}, \mathbf{x}^{\prime} \in X,
$$

where the norms are Euclidean norms on $\mathbb{R}^{s}$ and $\mathbb{R}^{n}$, respectively.

(2) The tiling of $\mathbb{R}^{n}$ by the Voronoi domains induced by $X$ (with the points of $X$ marked) contains finitely many translation-equivalence classes of marked Voronoi domains.

(3) The Delaunay tiling of $\mathbb{R}^{n}$ induced by $X$ contains finitely many translationequivalence classes of Delaunay domains.

In Section 2 we also show that Delone sets of finite type in $\mathbb{R}^{n}$ have bounds on their number of distinct local configurations under translations. Let $N_{X}^{*}(T)$ be the atlas counting function which counts the number of translation-inequivalent patches $(X-\mathbf{x}) \cap B(\mathbf{0} ; T)$ of radius $T$ over all $\mathbf{x} \in X$. The configurational entropy $H_{c}(X)$ of a set $X$ in $\mathbb{R}^{n}$ is defined by

$$
H_{c}(X):=\limsup _{T \rightarrow \infty} \frac{\log N_{X}^{*}(T)}{T^{n}}
$$

in analogy with definitions in [41]. We show that any Delone set of finite type $X$ has finite configurational entropy, i.e., there exists a constant $C$ depending on $X$ such that

$$
N_{X}^{*}(T) \leq \exp \left(C T^{n}\right)
$$

This is best possible; in Section 3 we give an example of a Meyer set $X$ in $\mathbb{R}^{n}$ with positive configurational entropy

$$
N_{X}^{*}(T) \geq \exp \left(C^{\prime} T^{n}\right) \quad \text { as } \quad T \rightarrow \infty,
$$

where $C^{\prime}$ is a positive constant.

In Section 3 we study Meyer sets viewed as a subclass of Delone sets of finite type. These sets have been extensively studied and characterized by Meyer [53] and Moody [54], [55]. We show that they are exactly those Delone sets $X$ of finite type whose address map is an approximate linear mapping on $X$. That is, they are those sets $X$ such that there exists a one-to-one linear map $\tilde{L}: \mathbb{R}^{n} \rightarrow \mathbb{R}^{s}$ such that

$$
\|\varphi(\mathbf{x})-\tilde{L}(\mathbf{x})\| \leq C, \quad \text { all } \quad \mathbf{x} \in X .
$$

Meyer previously showed that a set $X$ is a Meyer set if and only if every homomorphism $\psi:[X] \rightarrow \mathbb{R}^{d}$ for $1 \leq d<\infty$ is an approximate linear mapping on $X$, see Theorem 9.1 of [29]. Our small addition here is to observe that it suffices to check this property for the single map $\varphi$. The criterion (1.7) is useful in determining whether a set $X$ is a Meyer set, and we apply it in Section 5. We also show that every Meyer set $X$ is contained in some cut-and-project set $Y$ of dimension at most $\operatorname{rank}(X)$.

We introduce a relation of local derivability between Delone sets which parallels a notion for tilings introduced by Baake et al. [3]. Let $Y$ be a Delone set which is 
locally derivable from $X$. We show that if $X$ is a Delone set of finite type, then so is $Y$ (Corollary 2.1c), and if $X$ is a Meyer set, then so is $Y$ (Corollary 3.1b).

In Section 4 we study Delone sets $X$ that have an inflation symmetry $\eta$, which is a real number $\eta>1$ such that $\eta X \subseteq X$. This is not a true symmetry, since $X \backslash \eta X$ contains infinitely many points. Delone sets having an inflation symmetry can be obtained from self-similar tiling models of quasicrystals as sets of "control points," see [18], [32], [53], and [74]. We show that:

(1) If $X$ is a finitely generated Delone set, then $\eta$ is an algebraic integer.

(2) If $X$ is a Delone set of finite type, then $\eta$ is an algebraic integer all of whose algebraic conjugates $\eta^{\prime}$ satisfy $\left|\eta^{\prime}\right| \leq \eta$.

(3) If $X$ is a Meyer set, then $\eta$ is an algebraic integer all of whose algebraic conjugates $\eta^{\prime}$ satisfy $\left|\eta^{\prime}\right| \leq 1$.

The result (3) was originally proved by Meyer [53, Theorem 6]. These results are apparently best possible in the sense that if $\eta$ has the stated property, then there exists an appropriate Delone set $X$ having the symmetry $\eta X \subseteq X$. This has been demonstrated for cases (1) and (3), and has been checked for some extremal examples in case (2).

In Section 5 we study one-dimensional Delone sets of finite type. Such sets correspond exactly to tilings of the line $\mathbb{R}$ using a finite set of prototiles which are intervals of different lengths. We study special examples that are constructed using one-dimensional symbolic dynamical systems. These examples include some Meyer sets which might not be considered "quasicrystals." We also discuss diffraction spectra of one-dimensional sets. It is not known whether there exist (minimal) non-Meyer sets which contain some discrete component in their diffraction spectrum.

The deepest problem about quasicrystalline materials concerns finding physical mechanisms that lead to noncrystallographic ordered structures. ${ }^{3}$ This paper does not address this question. However, we note that many recent studies of quasicrystalline structure propose "clusters" or "local rules" as representing locally energy-minimizing finite arrangements of atoms at low temperature, and then propose decorations or "matching rules" for how clusters may overlap or abut each other as producing quasicrystalline structures, see [17], Section 2.2 of [23], Section 6 of [28], and [45]. The resulting structures are Delone sets of finite type.

The viewpoint of this paper is that Delone sets of finite type form a suitable class of sets that encompass all structures which might reasonably be called quasicrystalline. The narrower class of Meyer sets has previously been proposed as such a class [29][32]. However self-affine tilings of $\mathbb{R}^{n}$ which have an inflation matrix whose largest eigenvalue is not a Pisot or Salem number correspond (by picking "control points" in tiles) to Delone sets of finite type which are not Meyer sets. There exist such sets that have long-range order under translations in the sense of having a well-defined diffraction measure. That is, they have a unique autocorrelation measure in the sense of Hof [24], [26]; the diffraction measure is the Fourier transform of the autocorrelation measure. These diffraction measures are not known to contain any discrete component except at $\mathbf{0}$. It may be that there exist Delone sets of finite type which are not Meyer sets which have

\footnotetext{
${ }^{3}$ Even the problem of giving a physical explanation for crystallographic order is not resolved, see [63] and [65].
} 
the quasicrystalline property of having some "Bragg peaks" in their diffraction patterns; this is currently an open question, see Section 5.

Various special cases of the mathematical framework developed here are implicit in previous work. The notion of finite type for tilings appears under the name "finite number of local patterns" in [74, p. 699] where earlier references are cited. Robinson [67] defines a notion of "tiling of finite type" which corresponds to a tiling which satisfies some finite set of "local rules" under translations. Analogues of the address map have been previously introduced in special cases for tiling models for quasicrystals, in studying local rules for such tilings. For example, it appears as the "lifting function" in [45], see also [31] and [42]. For cut-and-project sets it appears as "perp-space coordinates" in Section 3.1.1 of [23]. Danzer [5] has long advocated the study of sets describable by local conditions. Danzer and Dolbilin [7] recently introduced a notion of Delone graph which is analogous to "local rules under isometries." They prove an analogue of Lemma 2.2, which is further sharpened in [6].

Note on Terminology. The concept of "quasicrystal" does not have a generally agreed on definition in the literature. In the physics literature [77] it typically refers to materials whose X-ray diffraction patterns exhibit some "Bragg peaks" with noncrystallographic symmetries; thus crystals are not considered quasicrystals. In this paper we do not define the term "quasicrystal" but the various classes of sets we define are inclusive: we view ideal crystals as a special kind of "quasicrystal." In the physics literature "long-range order" refers to the presence of Bragg peaks in a diffraction spectrum. Analogously to this we consider a Delone set $X$ to have strong long-range order under translations if $[X]$ is a quasilattice and $X$ has a well-defined diffraction measure in the sense of Hof [24], [26] which contains some discrete spectrum whose points span $\mathbb{R}^{n}$. We consider a Delone set $X$ to have long-range order under translations if $[X]$ is a quasilattice and $X$ has a well-defined diffraction measure, i.e., $X$ has a unique autocorrelation measure. Finally, we consider a Delone set $X$ to have weak long-range order under translations if $[X]$ is a quasilattice, i.e., if $X$ is a finitely generated Delone set; this is a mathematical notion of long-range order that may not be easily detectable physically.

Notation. $\|\cdot\|$ denotes the Euclidean norm on $\mathbb{R}^{n} . B(\mathbf{x} ; T)$ is the closed ball of radius $T$ around $\mathbf{x} \in \mathbb{R}^{n}$, i.e., $B(\mathbf{x} ; T):=\{\mathbf{y}:\|\mathbf{y}-\mathbf{x}\| \leq T\}$. The symbol $C$ designates a positive constant, which may be different at different occurrences.

\section{Delone Sets of Finite Type}

A Delone set $X$ is of finite type if $X-X$ is a discrete closed set, i.e., each ball $B(\mathbf{0} ; T)$ contains only finitely many points of $X-X$. In this section we characterize Delone sets of finite type in several ways and establish some of their properties.

Lemma 2.1. If $X$ is a Delone set of finite type, then any Delone set $Y$ with $Y \subseteq X$ is a Delone set of finite type. 
Proof. The set $Y-Y \subseteq X-X$, hence each ball $B(\mathbf{0} ; T)$ contains only finitely many points of $Y-Y$.

It is useful to have a quantitative measure of the discreteness of the closed set $X-X$.

Definition 2.1. For a Delone set $X$, its distance counting function $D_{X}(T)$ is

$$
D_{X}(T):=\#\{\mathbf{y} \in X-X:\|\mathbf{y}\| \leq T\} .
$$

A Delone set $X$ is of finite type if and only if $D_{X}(T)$ is finite for all $T>0$.

We now prove a "local rules" criterion for a Delone set to be of finite type, which also establishes that such sets are finitely generated.

Theorem 2.1. If $X$ is a Delone set with relative denseness constant $R$, then $X$ is a Delone set of finite type if and only if

$$
D_{X}(2 R)=\#\{(X-X) \cap B(\mathbf{0} ; 2 R)\} \quad \text { is finite. }
$$

Any Delone set $X$ of finite type is finitely generated, with

$$
\operatorname{rank}(X) \leq D_{X}(2 R) .
$$

To prove Theorem 2.1 we use the following "stepping-stone" property of Delone sets.

Lemma 2.2. Let $X$ be a Delone set in $\mathbb{R}^{n}$ with parameters $(r, R)$. Given any two points $\mathbf{x}, \mathbf{x}^{\prime} \in X$ there exists a chain of points

$$
\mathbf{x}=\mathbf{x}_{0}, \mathbf{x}_{1}, \mathbf{x}_{2}, \ldots, \mathbf{x}_{m}=\mathbf{x}^{\prime}
$$

in X such that

(i) $\left\|\mathbf{x}_{i}-\mathbf{x}_{i-1}\right\| \leq 2 R$, for $1 \leq i \leq m$.

(ii) $m \leq\left(4 R / r^{2}\right)\left\|\mathbf{x}-\mathbf{x}^{\prime}\right\|$.

Proof. We construct the sequence recursively as follows. Given $\mathbf{x}_{i}$, if $\left\|\mathbf{x}_{i}-\mathbf{x}^{\prime}\right\| \leq 2 R$ set $m=i+1$ and stop. Otherwise consider the closed ball

$$
B_{i}:=B\left(\mathbf{x}_{i}+R \frac{\mathbf{x}^{\prime}-\mathbf{x}_{i}}{\left\|\mathbf{x}^{\prime}-\mathbf{x}_{i}\right\|} ; R\right)
$$

of radius $R$ which has the property that $\mathbf{x}_{i}$ is on its boundary, and $\mathbf{x}_{i}$ is the furthest point in this ball from $\mathbf{x}^{\prime}$. This ball $B_{i}$ necessarily contains another point of $X$. To show this we consider for $\varepsilon>0$ the translated ball

$$
B_{i}(\varepsilon):=B\left(\mathbf{x}_{i}+(R+\varepsilon) \frac{\mathbf{x}^{\prime}-\mathbf{x}_{i}}{\left\|\mathbf{x}^{\prime}-\mathbf{x}_{i}\right\|} ; R\right) .
$$

It contains some point of $X$ by the Delone property, call it $\mathbf{x}(\varepsilon)$, and $\mathbf{x}(\varepsilon) \neq \mathbf{x}$ since $\mathbf{x}_{i} \notin B_{i}(\varepsilon)$ for $\varepsilon>0$. Since $X$ is discrete, some point occurs infinitely often among the 
points $\{\mathbf{x}(1 / n): n=1,2, \ldots\}$, call it $\mathbf{x}_{i+1}$, and it is in $B_{i}$ because its distance from $B_{i}$ is less than $1 / n$ for infinitely many $n$. Thus (i) holds. Next, the Delone property says that

$$
\left\|\mathbf{x}_{i+1}-\mathbf{x}_{i}\right\| \geq r
$$

However, $\mathbf{x}_{i}$ is the furthest point in the ball $B_{i}$ from $\mathbf{x}^{\prime}$, so this condition slices a spherical cap off $B_{i}$, and there is a constant $C$ such that

$$
\left\|\mathbf{x}_{i+1}-\mathbf{x}^{\prime}\right\| \leq\left\|\mathbf{x}_{i}-\mathbf{x}^{\prime}\right\|-C
$$

One can take $C=r^{2} / 4 R$, so (ii) follows.

Proof of Theorem 2.1. If $X$ is a Delone set of finite type, then (2.2) certainly holds.

To show the converse, suppose that (2.2) holds. Given $\mathbf{x}, \mathbf{x}^{\prime} \in X$, by Lemma 2.2 there exists a chain of points (2.4) in $X$ connecting $\mathbf{x}$ to $\mathbf{x}^{\prime}$ that satisfies conditions (i) and (ii). These conditions together imply that $(X-X) \cap B(\mathbf{0} ; T)$ is finite for any fixed $T$. To see this, note that the chain (2.4) connecting two points $\mathbf{x}, \mathbf{x}^{\prime} \in X$ with $\left\|\mathbf{x}-\mathbf{x}^{\prime}\right\| \leq T$ has at most $C T$ steps by (ii), and there are at most $D_{X}(2 R)$ choices for each step $\mathbf{x}_{i+1}-\mathbf{x}_{i}$ in the chain, by (i). Thus $D_{X}(T)=\#\{(X-X) \cap B(\mathbf{0}, T)\}$ is finite for all $T$, hence $X$ is of finite type.

Finally, the existence for all $\mathbf{x}^{\prime} \in X$ of the chain (2.4) satisfying (i) and (ii) shows that

$$
[X]:=\mathbb{Z}[X] \subseteq \mathbb{Z}\left[\mathbf{x}_{0} \cup\{\mathbf{x}: \mathbf{x} \in(X-X) \cap B(\mathbf{0} ; 2 R)\}\right] .
$$

Thus (2.3) follows.

The finiteness criterion of Theorem 2.1 carries over to finiteness of local neighborhoods of the set $X$ under translations.

Definition 2.2. (i) The T-neighborhood of a point $\mathbf{x} \in X$ is the set

$$
\mathcal{P}_{X}(\mathbf{x}, T):=X \cap B(\mathbf{x} ; T)=\left\{\mathbf{x}^{\prime} \in X:\left\|\mathbf{x}^{\prime}-\mathbf{x}\right\| \leq T\right\} .
$$

A $T$-patch of $X$ is a $T$-neighborhood of $X$ regarded as movable under translations.

(ii) A centered $T$-patch $\mathcal{P}$ is a $T$-neighborhood translated to the origin, i.e.,

$$
\mathcal{P}:=\mathcal{P}_{X}(\mathbf{x} ; T)-\mathbf{x}
$$

Definition 2.3. (i) The T-atlas $\mathcal{A}_{X}(T)$ of a Delone set $X$ is the set of all centered $T$-patches of $X$, i.e.,

$$
\mathcal{A}_{X}(T):=\{X \cap B(\mathbf{0} ; T)-\mathbf{x}: \mathbf{x} \in X\}
$$

(ii) The atlas counting function $N_{X}^{*}(T)$ counts the number of different centered $T$ patches, i.e.,

$$
N_{X}^{*}(T):=\left|\mathcal{A}_{X}(T)\right|, \quad \text { for } \quad T>0 .
$$


Corollary 2.1a. Let $X$ be a Delone set with relative denseness constant $R$. Then $X$ is a Delone set of finite type if and only if it has finitely many different centered $2 R$-patches, i.e., $N_{X}^{*}(2 R)<\infty$.

Proof. This follows from Theorem 2.1, since if $X$ has finitely many different $2 R$ patches then $D_{X}(2 R)$ is finite, while if $D_{X}(2 R)$ is finite, then there are finitely many choices for points within distance $2 R$ of a fixed point $\mathbf{x}$.

Delone sets of finite type arise from tilings of $\mathbb{R}^{n}$ by translates of a finite nonempty set of prototiles. We suppose that each prototile is simply connected, is the closure of its interior, and has a boundary of measure zero. To any such tiling $\mathcal{T}$ we can associate a Delone set (in many different ways) by marking a finite set of points in each prototile, and taking $X$ to be the set of marked points in the tiling. We say that such a tiling $\mathcal{T}$ has a finite number of local patches if it contains only finitely many translation-inequivalent arrangements of neighbors of each prototile. We have:

Corollary 2.1b. Let $\mathcal{T}$ be a tiling of $\mathbb{R}^{n}$ by translations of a finite set of prototiles. Suppose that each prototile is decorated by a finite nonempty set of points, and let $X=X(\mathcal{T})$ be the point set associated to $\mathcal{T}$ by these decorations. If $\mathcal{T}$ has a finite number of local patches, then $X(\mathcal{T})$ is a Delone set of finite type, and conversely.

Proof. This follows from Theorem 2.1 because there are only finitely many translationinequivalent ways to tile neighborhoods of diameter $2 R$.

Baake et al. [3] (see also [1] and [2]) introduced a notion of "local derivability" between tilings, which has the following analogue for Delone sets.

Definition 2.4. (i) Let $X$ and $Y$ both be Delone sets which are relatively dense with constant $R$. We say that $Y$ is locally derivable from $X$ if there is a constant $R^{\prime}$ such that for each $\mathbf{x} \in X$ the set of points of $Y \cap B(\mathbf{x} ; 2 R)$ is completely determined by the centered $R^{\prime}$-patch of $X$ around $\mathbf{x}$. That is, the point set $\mathcal{P}_{\mathbf{x}}(Y ; 2 R)=(Y \cap B(\mathbf{x} ; R))-\mathbf{x}$ is a function of the centered patch $\mathcal{P}_{\mathbf{x}}\left(X, R^{\prime}\right)=\left(X \cap B\left(\mathbf{x} ; R^{\prime}\right)\right)-\mathbf{x}$.

(ii) We say that $X$ and $Y$ are mutually locally derivable if each is locally derivable from the other.

Corollary 2.1c. If $X$ is a Delone set of finite type and $Y$ is a Delone set locally derivable from $X$, then $Y$ is a Delone set of finite type.

Proof. By hypothesis $D_{X}\left(R^{\prime}\right)$ is finite. Since the translation classes of $R^{\prime}$-patches of $\mathrm{X}$ determine the $2 R$-patches of $Y$, we have $D_{Y}(2 R)$ is finite, and Theorem 2.1 applies.

Our next object is to derive several equivalent criteria for a set $X$ to be a Delone set of finite type. To formulate these, we make some definitions. 
Definition 2.5. A Delone set $X$ has the locally finite atlas property under translations if $N_{X}^{*}(T)$ is finite for all $T>0$.

Definition 2.6. (i) Given a discrete set $X$ in $\mathbb{R}^{n}$ and a point $\mathbf{x} \in X$ the Voronoi domain $V_{X}(\mathbf{x})$ at $\mathbf{x}$ is the set of all points in $\mathbb{R}^{n}$ as close to $\mathbf{x}$ as to any other point of $X$, namely,

$$
V_{X}(\mathbf{x})=\left\{\mathbf{y}:\|\mathbf{y}-\mathbf{x}\| \leq\left\|\mathbf{y}-\mathbf{x}^{\prime}\right\|, \text { all } \mathbf{x}^{\prime} \in X\right\} .
$$

A marked Voronoi domain is a Voronoi domain together with a specific point in it viewed as a marked point.

(ii) The set of Voronoi domains of a discrete set $X$ form a tiling of $\mathbb{R}^{n}$ called the Voronoi tesselation of $\mathbb{R}^{n}$ induced by $X$. A marked Voronoi tesselation consists of the Voronoi tesselation induced by a discrete set $X$ with the points of $X$ marked.

If $X$ is a Delone set, then each Voronoi domain $V_{X}(\mathbf{x})$ is a bounded convex polytope contained in $B(\mathbf{x} ; 2 R)$. (For a general discrete set $X$ the set $V_{X}(\mathbf{x})$ is a convex polytope, possibly unbounded.) The set $X$ cannot always be uniquely reconstructed from its Voronoi tesselation. It is uniquely determined by the induced Voronoi tesselation plus a single Voronoi domain marked with a point of $X$; this determines the marking in all other Voronoi domains by the points of $X$.

Definition 2.7. (i) Given a Delone set $X$ in $\mathbb{R}^{n}$ a finite subset $Y$ of $X$ determines an empty sphere if all points of $Y$ lie on a sphere in $\mathbb{R}^{n}$, there are no points of $X$ in the interior of this sphere, and the convex hull of $Y$ has nonempty interior. We call the convex hull of all points of $X$ lying on this sphere the Delaunay domain determined by $Y$ in $X$. It is a convex polytope.

(ii) The set of Delaunay domains of a Delone set $X$ give a tiling of $\mathbb{R}^{n}$ called the Delaunay tesselation of $\mathbb{R}^{n}$ induced by $X$.

The Delaunay tesselation was introduced by Delone [9] in 1934. He showed that for a Delone set $X$ in "general position" all Delaunay domains are simplices; thus the Delaunay tesselation is often called the Delaunay triangulation in the computational geometry literature, ${ }^{4}$ see p. 49 of [58]. A proof that Delaunay domains give a facet-tofacet tiling of $\mathbb{R}^{n}$ can be found in Fortune [15], and in the "generic case" in Chapter 8 of [68]. The set $X$ is uniquely reconstructible from its associated Delaunay tesselation, because $X$ is the set of all vertices of all Delaunay domains. For general references on Voronoi and Delaunay tesselations, see [14] and [15].

Theorem 2.2. For a Delone set $X$ in $\mathbb{R}^{n}$, the following properties are equivalent:

(i) $X$ is a Delone set of finite type.

(ii) $X$ has the locally finite atlas property under translations.

\footnotetext{
${ }^{4}$ The spelling Delaunay triangulation is standard in the computational geometry literature. Here "general position" means that there do not exist $n+2$ points of $X$ lying on any sphere of diameter at most $2 R$. Rogers [68, Chapter 8] requires instead that the set $X$ have no $n+2$ equidistant points; in this case a Delaunay domain as we have defined it can be triangulated in a canonical way to obtain his "Delaunay triangulation."
} 
(iii) The marked Voronoi tesselation of $\mathbb{R}^{n}$ induced by $X$ has finitely many translationinequivalent marked Voronoi domains.

(iv) The Delaunay tesselation of $\mathbb{R}^{n}$ induced by $X$ has finitely many translationinequivalent Delaunay domains.

(v) $X$ is finitely generated and any address map $\varphi:[X] \rightarrow \mathbb{Z}^{s}$ has

$$
\left\|\varphi(\mathbf{x})-\varphi\left(\mathbf{x}^{\prime}\right)\right\| \leq C_{0}\left\|\mathbf{x}-\mathbf{x}^{\prime}\right\|, \quad \text { all } \quad \mathbf{x}, \mathbf{x}^{\prime} \in X,
$$

for some constant $C_{0}$ depending on $\varphi$.

Proof. (i) $\Rightarrow$ (ii) Since $(X-X) \cap B(\mathbf{0} ; T)$ is finite, and for $\mathbf{x} \in X$ all points in

$$
\mathcal{P}(\mathbf{x} ; T):=(X-\mathbf{x}) \cap B(0 ; T)
$$

lie in this set, there are only finitely many choices for $\mathcal{P}(\mathbf{x} ; T)$. In fact

$$
N_{X}^{*}(T) \leq 2^{D_{X}(T)} .
$$

(ii) $\Rightarrow$ (iii) A marked Voronoi domain $V_{X}(\mathbf{x})$ is completely determined by the patch

$$
\mathcal{P}(\mathbf{x} ; 2 R):=(X-\mathbf{x}) \cap B(\mathbf{0} ; 2 R) .
$$

Any point $\mathbf{y}$ with $\|\mathbf{x}-\mathbf{y}\|>2 R$ is within distance $2 R$ of some point $\mathbf{x}^{\prime} \in X$, hence cannot be in $V_{X}(\mathbf{x})$. The locally finite atlas property says that there are only $N_{X}^{*}(2 R)<\infty$ such patches, hence there are at most $N_{X}^{*}(2 R)$ possible translation-inequivalent Voronoi domains.

(iii) $\Rightarrow$ (i) The Delone property of $X$ guarantees that the ball $B\left(\mathbf{x} ; \frac{1}{2} r\right)$ is contained in the Voronoi domain $V_{X}(\mathbf{x})$. The facets of each marked Voronoi domain determine the marking in each neighboring Voronoi domain, namely, drop a perpendicular from $\mathbf{x}$ to the hyperplane determined by the facet and extend it an equal distance to the other side. By hypothesis there are only a finite number of choices for each neighboring domain, up to translation. Continue adding marked Voronoi domains in this way to fill up the entire ball of radius $2 R$ around $\mathbf{x}$. One has to add at most $2 R / r$ concentric layers of Voronoi domains around $\mathbf{x}$ to do this. There are only finitely many possible extensions, and this locates all possible locations for elements of $X$ within distance $2 R$ of $X$. Since this construction is translation-invariant, we conclude that $(X-X) \cap B(\mathbf{0} ; 2 R)$ is finite. Now (i) follows by Theorem 2.1.

(i) $\Rightarrow$ (iv) A Delaunay domain including a given point $\mathbf{x} \in X$ is completely determined by all points within distance $2 R$ of $X$, since the largest empty sphere touching $X$ has diameter at most $2 R$. By hypothesis (i) there are only finitely many translation-inequivalent arrangements of such points.

(iv) $\Rightarrow$ (i) The Delaunay tesselation of $X$ uniquely determines $X$. Given any two elements of $X$ within a distance $2 R$, there is a path of edges in the Delaunay tesselation connecting them which remains in a ball of radius $4 R$ about one of the points. Since a Delaunay tesselation meets vertex-to-vertex, hypothesis (iv) implies that there are only finitely many ways to tile a ball of radius $4 R$. Thus $D_{X}(2 R)$ is finite, and Theorem 2.1 implies that $X$ is a Delone set of finite type. 
(i) $\Rightarrow$ (v) A Delone set $X$ of finite type is finitely generated by Theorem 2.1. To show the other part, suppose that $\varphi:[X] \rightarrow \mathbb{Z}^{s}$ is an address map and set

$$
C_{2}:=\max \{\|\varphi(\mathbf{y})\|: \mathbf{y} \in(X-X) \cap B(0 ; 2 R)\} .
$$

Given $\mathbf{x}, \mathbf{x}^{\prime} \in X$ by Lemma 2.2 there exists a chain (2.4) at points of $X$ connecting $\mathbf{x}$ to $\mathbf{x}^{\prime}$ that satisfies $\left\|\mathbf{x}_{i+1}-\mathbf{x}_{i}\right\| \leq 2 R$ and the number of points is at most $\left(4 R / r^{2}\right)\left\|\mathbf{x}_{i+1}-\mathbf{x}_{i}\right\|$. Using the linearity of $\varphi$ on $[X]$, we have

$$
\begin{aligned}
\left\|\varphi(\mathbf{x})-\varphi\left(\mathbf{x}^{\prime}\right)\right\| & \leq \sum_{i=1}^{m}\left\|\varphi\left(\mathbf{x}_{i+1}\right)-\varphi\left(\mathbf{x}_{i}\right)\right\| \\
& =\sum_{i=1}^{m}\left\|\varphi\left(\mathbf{x}_{i+1}-\mathbf{x}_{i}\right)\right\| \\
& \leq C_{2} m \leq \frac{4 C_{2} R}{r^{2}}\left\|\mathbf{x}-\mathbf{x}^{\prime}\right\|,
\end{aligned}
$$

which proves (iv) with $C_{0}=4 C_{2} R / r^{2}$.

(v) $\Rightarrow$ (i) If $\mathbf{x}, \mathbf{x}^{\prime} \in X$ satisfy $\left\|\mathbf{x}^{\prime}-\mathbf{x}\right\| \leq 2 R$, then by hypothesis

$$
\left\|\varphi\left(\mathbf{x}-\mathbf{x}^{\prime}\right)\right\|=\left\|\varphi(\mathbf{x})-\varphi\left(\mathbf{x}^{\prime}\right)\right\| \leq 2 C R .
$$

Addresses all lie in $\mathbb{Z}^{s}$, hence there are only finitely many choices for $\varphi\left(\mathbf{x}-\mathbf{x}^{\prime}\right)$, hence for $\mathbf{x}-\mathbf{x}^{\prime}$, since $\varphi: X \rightarrow \mathbb{Z}^{s}$ is one-to-one. Thus $(X-X) \cap B(\mathbf{0} ; 2 R)$ is finite, so $X$ is of finite type by Theorem 2.1 .

Theorem 2.2 yields an upper bound on the growth of the distance counting function $D_{T}(X)$.

Corollary 2.2. Let $X$ be a Delone set of finite type, of rank s. There exists a positive constant $C_{1}$ depending on $X$ such that

$$
D_{X}(T) \leq C_{1} T^{s}, \quad \text { all } \quad T \geq 1 .
$$

Proof. Suppose that $\mathbf{x}, \mathbf{x}^{\prime} \in X$ with $\left\|\mathbf{x}-\mathbf{x}^{\prime}\right\| \leq T$. The point $\mathbf{x}-\mathbf{x}^{\prime}$ is determined by its address $\varphi\left(\mathbf{x}-\mathbf{x}^{\prime}\right)$, and, by (iv) of Theorem 2.2, there is a constant $C_{0}$ such that

$$
\left\|\varphi\left(\mathbf{x}-\mathbf{x}^{\prime}\right)\right\| \leq C_{0}\left\|\mathbf{x}-\mathbf{x}^{\prime}\right\|=C_{0} T .
$$

The set of elements in $\mathbb{Z}^{s}$ in a ball of radius $T$ is at most $C T^{s}$ for all $T \geq 1$, and (2.12) follows.

We next obtain an upper bound on the atlas counting function $N_{X}^{*}(T)$ of a Delone set of finite type.

Definition 2.8. The configurational entropy $H_{c}(X)$ of a Delone set $X$ in $\mathbb{R}^{n}$ is given by

$$
H_{c}(X):=\limsup _{T \rightarrow \infty} \frac{\log N_{X}^{*}(T)}{T^{n}} .
$$


We prove:

Theorem 2.3. If $X$ is a Delone set of finite type in $\mathbb{R}^{n}$, there is a constant $C_{2}$ depending on $X$ such that

$$
N_{X}^{*}(T) \leq \exp \left(C_{2} T^{n}\right), \quad \text { all } \quad T \geq 1 .
$$

That is, $X$ has finite configurational entropy.

Remark. The factor $T^{n}$ in the bound (2.13) cannot be improved, see Example 3.1 in Section 3.

Proof. We may build up all possible patches of radius $T$ out of cubical patches of the form $X \cap C(\mathbf{x} ; 8 R)$ for $\mathbf{x} \in X$, where $C(\mathbf{x} ; \rho)$ denotes a cube of side $2 \rho$ centered at $\mathbf{x}$. Since $X \cap C(\mathbf{x} ; 8 R) \subseteq X \cap B(\mathbf{x} ; 8 \sqrt{n} R)$, the number $C^{\prime}$ of translation-inequivalent cubical patches $X \cap C(\mathbf{x} ; 4 R)$ is at most $D_{X}(8 \sqrt{n} R)$.

We bound the number of different cubical patches of side $2 T$ centered at $\mathbf{0}$, all of those subpatches of side $2 R$ match those in the list $\mathcal{L}$. To begin, there are $C^{\prime}$ choices for the patch centered at $\mathbf{0}$. Now we proceed to add cubical patches in concentric layers. The centers of the new patches are chosen at already constructed points, within distance $R$ of the boundary, so the new cubical patch extends at least $3 R$ outside. At the $k$ th stage we have covered a cube of side $8+6 k R$. We can arrange that all cube centers chosen are separated by at least $2 R$, hence there are at most $O\left((T / R)^{n}\right)$ cubical patches to choose in the process. Thus there are at most $\left(C^{\prime}\right)^{C^{\prime \prime} T^{n}}$ possible choices of cubical patches of side $2 T$, which proves (2.13).

We conclude this section with an example showing that the requirement that $X-X$ be closed is necessary in the definition of a Delone set of finite type. The Delone set

$$
X=\left\{n+\frac{1}{3 n^{2}+3}: n \in \mathbb{Z}\right\}
$$

in $\mathbb{R}$ has the property that $X-X$ is discrete. However, $X-X$ has 1 as a finite limit point, so $X$ is not a Delone set of finite type; it is not even finitely generated.

\section{Meyer Sets}

Meyer [53] recently defined a mathematical notion which he called a "quasicrystal," which is based on concepts in harmonic analysis that he introduced around 1970, see [51] and [52]. These sets are termed Meyer sets by Moody [54], [55], who unifies and extends the known characterizations of a Meyer sets. Moody [55] takes a harmonic analysis criterion as the basic definition of a Meyer set (relatively dense harmonious set), while Meyer [53] defines them to be those Delone sets $X$ such that $X-X \subseteq X+F$ for some finite set $F$. These properties are equivalent to $X-X$ being a Delone set, see [38].

It is known that Meyer sets can also be characterized in terms of cut-and-project sets. 
Definition 3.1. Let $\Lambda$ be a full rank lattice in $\mathbb{R}^{d}=\mathbb{R}^{n+m}=\mathbb{R}^{n} \times \mathbb{R}^{m}$, and let $\pi^{\|}$and $\pi^{\perp}$ be orthogonal projections onto the factors $\mathbb{R}^{n}$ and $\mathbb{R}^{m}$, respectively. A window $\Omega$ is a bounded open subset of $\mathbb{R}^{m}$, and the strip $S(\Omega)$ in $\mathbb{R}^{d}$ associated to the window $\Omega$ is

$$
S(\Omega):=\mathbb{R}^{n} \times \Omega=\left\{\mathbf{w} \in \mathbb{R}^{d}: \pi^{\perp}(\mathbf{w}) \in \Omega\right\} .
$$

The cut-and-project set $X(\Lambda, \Omega)$ associated to the data $(\Lambda, \Omega)$ is

$$
X(\Lambda, \Omega)=\pi^{\|}(\Lambda \cap S(\Omega)) .
$$

We call $d$ the dimension of the data $(\Lambda, \Omega)$. A given cut-and-project set $X$ may be constructed in many ways, using different pairs $(\Lambda, \Omega)$ and $\left(\Lambda^{\prime}, \Omega^{\prime}\right)$ of different dimensions. (Some authors allow the window $\Omega$ to be a closed set, but we do not.)

An alternate definition of cut-and-project sets, which is used in [24] and [59], appears slightly different but gives the same sets. It takes a fixed lattice $\mathbb{Z}^{d}$ in $\mathbb{R}^{d}$, and projects it onto an arbitrary pair $\left(E^{\|}, E^{\perp}\right)$ of orthogonal subspaces of dimensions $n$ and $m$, respectively, using orthogonal projections $\pi^{\|}$and $\pi^{\perp}$, respectively. It identifies $E^{\|}$with $\mathbb{R}^{n}$ by a linear map, and takes a window set $\Omega$ in $E^{\perp}$.

Definition 3.2. A cut-and-project set is nondegenerate if $\pi^{\|}: \mathbb{R}^{d} \rightarrow \mathbb{R}^{n}$ is one-to-one on $\Lambda$, i.e., if

$$
\Lambda \cap\left(\{\mathbf{0}\} \times \mathbb{R}^{m}\right)=\{(\mathbf{0}, \mathbf{0})\} .
$$

It is irreducible if it is nondegenerate and $\pi^{\perp}(\Lambda)$ is dense in $\mathbb{R}^{m}$.

An irreducible cut-and-project set coincides with the concept of a model set of Meyer [52]. Meyer proved that a Delone set $X$ is a Meyer set if and only if there is an irreducible cut-and-project set $X^{\prime}$ and a finite set $F$ such that

$$
X \subseteq X^{\prime}+F^{\prime}
$$

see [52], [53], and [55].

Meyer sets are Delone sets of finite type, hence are finitely generated. We characterize which finitely generated Delone sets are Meyer sets in terms of their address maps, using the following concept due to Meyer.

Definition 3.3. A map $\psi:[X] \rightarrow \mathbb{R}^{d}$ is an almost linear mapping on $X$ if there exists a linear map $L_{\psi}: \mathbb{R}^{n} \rightarrow \mathbb{R}^{d}$ such that

$$
\left\|\psi(\mathbf{x})-L_{\psi}(\mathbf{x})\right\|<C, \quad \text { all } \quad \mathbf{x} \in X .
$$

Condition (3.3) applies only to $\mathbf{x} \in X$ and not to general elements of $[X]$.

The following result gives several characterizations of Meyer sets. These characterizations are mainly due to Meyer (see also [55]); the equivalence of (iv) and (v) seems to be new. 
Theorem 3.1. The following properties of a discrete set $X$ in $\mathbb{R}^{n}$ are equivalent:

(i) $X$ is a Meyer set. That is, $X-X$ is a Delone set.

(ii) $X$ is a Delone set and there is a finite set $F$ such that

$$
X-X \subseteq X+F .
$$

(iii) $X$ is a finitely generated Delone set and every homomorphism $\psi:[X] \rightarrow \mathbb{R}^{d}$ for some $d \geq 1$ is an almost linear mapping on $X$.

(iv) $X$ is a finitely generated Delone set and the address map $\varphi:[X] \rightarrow \mathbb{Z}^{s}$ is an almost linear mapping on $X$, i.e., there is a linear map $\tilde{L}: \mathbb{R}^{n} \rightarrow \mathbb{R}^{s}$ and a constant $C$ such that

$$
\|\varphi(\mathbf{x})-\tilde{L}(\mathbf{x})\| \leq C, \quad \text { all } \quad \mathbf{x} \in X .
$$

(v) $X$ is a finitely generated Delone set and there exists a nondegenerate cut-andproject set $X^{\prime}$ of dimension at most $\operatorname{rank}(X)$ such that

$$
X \subseteq X^{\prime}
$$

Remark. The cut-and-project set $X^{\prime}$ containing $X$ that appears in (v) is not necessarily irreducible.

Proof. (i) $\Leftrightarrow$ (ii) This is proved in [38].

(ii) $\Rightarrow$ (iii) This result is due to Meyer and a proof can be found in Section 8 of [55]. We mention some important points. The map $\tilde{L}: \mathbb{R}^{n} \rightarrow \mathbb{R}^{s}$ can be constructed as an "ideal address map," as follows. For each $\mathbf{y} \in \mathbb{R}^{n}$ define

$$
\tilde{L}(\mathbf{y}):=\lim _{k \rightarrow \infty} \frac{\varphi\left(\mathbf{x}_{k}\right)}{2^{k}},
$$

where $\mathbf{x}_{k} \in X$ satisfies $\left\|\mathbf{x}_{k}-2^{k} \mathbf{y}\right\| \leq R$. Using the Meyer set property one proves that this limit exists and is unique, and satisfies

$$
\tilde{L}\left(c \mathbf{y}+\mathbf{y}^{\prime}\right)=c \tilde{L}(\mathbf{y})+\tilde{L}\left(\mathbf{y}^{\prime}\right), \quad \mathbf{y}, \mathbf{y}^{\prime} \in \mathbb{R}^{n},
$$

hence is linear. We call $\tilde{L}$ an ideal address map because it satisfies the identity

$$
\mathbf{y}=\sum_{j=1}^{s} \tilde{L}(\mathbf{y})_{j} \mathbf{v}_{j}, \quad \text { all } \quad \mathbf{y} \in \mathbb{R}^{n} .
$$

This identity is derived from (3.6) using the address identity

$$
\mathbf{x}=\sum_{j=1}^{s} \varphi(\mathbf{x})_{j} \mathbf{v}_{j}, \quad \text { all } \quad \mathbf{x} \in[X] .
$$

(iii) $\Rightarrow$ (iv) Immediate.

(iv) $\Rightarrow$ (ii) Given $\mathbf{x}, \mathbf{x}^{\prime} \in X$, hypothesis (iv) gives

$$
\left\|\tilde{L}\left(\mathbf{x}-\mathbf{x}^{\prime}\right)-\varphi\left(\mathbf{x}-\mathbf{x}^{\prime}\right)\right\| \leq C .
$$


Since $X$ is a Delone set, there is some point $\mathbf{x}^{\prime \prime} \in X$ with

$$
\left\|\mathbf{x}^{\prime \prime}-\left(\mathbf{x}+\mathbf{x}^{\prime}\right)\right\| \leq R
$$

and by hypothesis

$$
\left\|\tilde{L}\left(\mathbf{x}^{\prime \prime}\right)-\varphi\left(\mathbf{x}^{\prime \prime}\right)\right\| \leq C
$$

Now

$$
\left\|\tilde{L}\left(\mathbf{x}^{\prime \prime}-\mathbf{x}+\mathbf{x}^{\prime}\right)\right\| \leq C^{\prime}\left\|\mathbf{x}^{\prime \prime}-\left(\mathbf{x}+\mathbf{x}^{\prime}\right)\right\| \leq C^{\prime} R,
$$

where $C^{\prime}=\|\tilde{L}\|$. The triangle inequality applied to (3.8)-(3.10) gives

$$
\left\|\varphi\left(\mathbf{x}^{\prime \prime}-\left(\mathbf{x}-\mathbf{x}^{\prime}\right)\right)\right\|=\left\|\varphi\left(\mathbf{x}^{\prime \prime}\right)-\varphi\left(\mathbf{x}-\mathbf{x}^{\prime}\right)\right\| \leq 2 C+C^{\prime} R,
$$

hence there are a finite number of choices for addresses in $\mathbb{Z}^{s}$ satisfying (3.11). Since the address map is one-to-one on $[X]$, there are a finite number of $\mathbf{y}=\mathbf{x}^{\prime \prime}-\left(\mathbf{x}-\mathbf{x}^{\prime}\right) \in[X]$ satisfying (3.11), and we call this set $F$. We have shown that

$$
X-X \subseteq X+F
$$

which proves (ii).

(iv) $\Rightarrow$ (v) We use the almost linear mapping $\tilde{L}: \mathbb{R}^{n} \rightarrow \mathbb{R}^{s}$ associated to the address map by (3.7) to construct a suitable nondegenerate cut-and-project set. Set $s=n+m$, so $m \geq 0$, and let the vector space

$$
V:=\left\{\tilde{L}(\mathbf{x}): \mathbf{x} \in \mathbb{R}^{n}\right\}
$$

be the image of the map $\tilde{L}$. We first show that $\operatorname{dim}(V)=n$. Define $\psi: \mathbb{R}^{s} \rightarrow \mathbb{R}^{n}$ by

$$
\psi\left(u_{1}, \ldots, u_{s}\right)=\sum_{j=1}^{s} u_{j} \mathbf{v}_{j}
$$

and observe that (3.7) gives

$$
\psi \circ \tilde{L}(\mathbf{y})=\mathbf{y} \quad \text { all } \quad \mathbf{y} \in \mathbb{R}^{n} .
$$

Thus $\psi(V)=\mathbb{R}^{n}$ hence $\operatorname{dim}(V)=n$. Now set

$$
W:=\operatorname{ker}(\psi)=\left\{\left(\ell_{1}, \ldots, \ell_{s}\right): \sum_{j=1}^{s} \ell_{j} \mathbf{v}_{j}=0\right\}
$$

Now $\operatorname{dim}(W)=s-n=m$. Choose an orthonormal basis of $W$,

$$
\ell^{(i)}=\left(\ell_{i 1}, \ldots, \ell_{i s}\right), \quad 1 \leq i \leq m,
$$

and set

$$
\mathbf{w}_{j}:=\tilde{L}\left(\mathbf{v}_{j}\right)-\mathbf{e}_{j}, \quad 1 \leq j \leq s,
$$


where $\mathbf{e}_{j}=(0, \ldots, 1, \ldots, 0)$ is the $j$ th coordinate vector. Now (3.7) implies that $\mathbf{w}_{j} \in$ $W$, hence we may define coefficients $\left\{\alpha_{j i}\right\}$ by

$$
\mathbf{w}_{j}=\sum_{i=1}^{m} \alpha_{j i} \ell^{(i)}, \quad 1 \leq j \leq s .
$$

Let $\Lambda=\mathbb{Z}\left[\mathbf{v}_{1}^{*}, \ldots, \mathbf{v}_{s}^{*}\right]$ be the additive subgroup of $\mathbb{R}^{s}$ spanned by

$$
\mathbf{v}_{j}^{*}:=\left(\mathbf{v}_{j}, \alpha_{j 1}, \alpha_{j 2}, \ldots, \alpha_{j m}\right), \quad 1 \leq j \leq s .
$$

We claim that $\Lambda$ is a full rank lattice in $\mathbb{R}^{d}$. To show this, it suffices to prove that the vectors $\mathbf{v}_{j}^{*}$ are $\mathbb{R}$-linearly independent. So suppose that

$$
\sum_{j=1}^{s} u_{j} \mathbf{v}_{j}^{*}=\mathbf{0}
$$

The first $n$ coordinates give

$$
\sum_{j=1}^{s} u_{j} \mathbf{v}_{j}=\mathbf{0},
$$

hence $\mathbf{u}=\left(u_{1}, \ldots, u_{d}\right) \in W$. Now the last $m$ coordinates give

$$
\sum_{j=1}^{s} u_{j} \alpha_{j i}=0, \quad 1 \leq i \leq m
$$

Thus

$$
\sum_{j=1}^{s} u_{j} \mathbf{w}_{j}=\sum_{j=1}^{s} u_{j}\left(\sum_{i=1}^{m} \alpha_{j i} \ell^{(i)}\right)=\sum_{i=1}^{m} \ell^{(i)}\left(\sum_{j=1}^{s} u_{j} \alpha_{j i}\right)=\mathbf{0},
$$

and the definition of $\mathbf{w}_{j}$ yields

$$
\sum_{j=1}^{s} u_{j} \tilde{L}\left(\mathbf{v}_{j}\right)-\left(u_{1}, u_{2}, \ldots, u_{s}\right)=\mathbf{0} .
$$

Since each coordinate is linear,

$$
\left(u_{1}, \ldots, u_{s}\right)=\tilde{L}\left(\sum_{j=1}^{s} u_{j} \mathbf{v}_{j}\right)=\tilde{L}(\mathbf{0})=\mathbf{0} .
$$

Thus $\left[\mathbf{v}_{1}^{*}, \ldots, \mathbf{v}_{s}^{*}\right]$ are linearly independent, and $\Lambda$ is a full rank lattice in $\mathbb{R}^{s}$.

The projection $\pi^{\|}: \mathbb{R}^{s} \rightarrow \mathbb{R}^{n}$ sends

$$
\pi^{\|}\left(\mathbf{w}_{j}\right)=\mathbf{v}_{j}, \quad 1 \leq j \leq s,
$$

hence it is one-to-one on $\Lambda$ since $[X]=\mathbb{Z}\left[\mathbf{v}_{1}, \ldots, \mathbf{v}_{s}\right]$ is a free module. 
Now suppose that $\mathbf{x} \in[X]$ and its address is

$$
\mathbf{x}=\sum_{j=1}^{s} \varphi(\mathbf{x})_{j} \mathbf{v}_{j}, \quad \text { with } \quad \varphi(\mathbf{x})_{j} \in \mathbb{Z} .
$$

Then

$$
\mathbf{x}^{*}:=\sum_{j=1}^{s} \varphi(\mathbf{x})_{j} \mathbf{w}_{j}
$$

has $\mathbf{x}^{*} \in \Lambda$, and $\pi^{\|}\left(\mathbf{x}^{*}\right)=\mathbf{x}$. Now set

$$
\pi^{\perp}\left(\mathbf{x}^{*}\right)=\sum_{j=1}^{s} \varphi(\mathbf{x})_{j}\left(\alpha_{j 1}, \alpha_{j 2}, \ldots, \alpha_{j m}\right)=\left(z_{1}, \ldots, z_{m}\right) .
$$

Since the vectors $\ell^{(i)}$ are orthonormal, we have

$$
\begin{aligned}
\left\|\pi^{\perp}\left(\mathbf{x}^{*}\right)\right\| & =\left(\sum_{j=1}^{m} z_{i}^{2}\right)^{1 / 2}=\left\|\sum_{i=1}^{m} z_{i} \ell^{(i)}\right\| \\
& =\left\|\sum_{i=1}^{m} \sum_{j=1}^{s} \varphi(\mathbf{x})_{j} \alpha_{j i} \ell^{(i)}\right\| \\
& =\left\|\sum_{j=1}^{s} \varphi(\mathbf{x})_{j} \mathbf{w}_{j}\right\| \\
& =\|\varphi(\mathbf{x})-\tilde{L}(\mathbf{x})\|,
\end{aligned}
$$

where (3.12) and (3.13) were used at the last step. The almost linear property (3.4) then yields

$$
\left\|\pi^{\perp}\left(\mathbf{x}^{*}\right)\right\| \leq C .
$$

It therefore suffices to take the window set

$$
\Omega=\left\{\mathbf{z} \in \mathbb{R}^{m}:\|\mathbf{z}\|<C+1\right\}
$$

to obtain a nondegenerate cut-and-project set $X(\Lambda, \Omega)$ of dimension $s$ with

$$
X \subseteq X(\Lambda, \Omega)
$$

which is $(\mathrm{v})$.

(v) $\Rightarrow$ (iv) This is a result of Meyer, for which see Theorem 9.1 of [55]. Alternatively one can directly reconstruct the map $\tilde{L}(\mathbf{x})$ from $X^{\prime}$, using

$$
\tilde{L}(\mathbf{x}):=\left(\pi^{\|}\right)^{-1}(\mathbf{x}) \cap \Lambda \quad \text { for } \quad \mathbf{x} \in X .
$$

This is well defined since $X^{\prime}$ is nondegenerate. Now $\tilde{L}$ extends to a linear map on $[X]$, and (3.4) holds by the cut-and-project property of $X^{\prime}$. 
The following result of Meyer follows directly from property (v) above.

Corollary 3.1a. If $X$ is a Meyer set and $Y$ is a Delone set with $Y \subseteq X$, then $Y$ is a Meyer set.

We obtain a similar result for sets locally derivable from Meyer sets.

Corollary 3.1b. If $X$ is a Meyer set and $Y$ is a Delone set locally derivable from $X$, then $Y$ is a Meyer set.

Proof. We claim that $Y \subseteq X+F^{\prime}$ for some finite set $F^{\prime}$. Indeed there are only finitely many different $R^{\prime}$-patches of $X$, and the elements of $Y$ can be viewed as obtained by individually shifting the center of each $R^{\prime}$-patch of $X$, in a finite number of different ways, so the claim follows. Since $X$ is a Meyer set, the set $X^{\prime}=X+F^{\prime}$ is a Meyer set because it satisfies property (ii) above:

$$
X^{\prime}-X^{\prime} \subseteq X^{\prime}+\left(F+F^{\prime}-F^{\prime}\right) .
$$

Now Y is a Meyer set by Corollary 3.1a.

We also apply Corollary 3.1a in the following example.

Example 3.1. There exists a Meyer set $X \subseteq \mathbb{Z}^{n}$ in $\mathbb{R}^{n}$ such that

$$
N_{X}^{*}(T) \geq \exp \left(C_{3} T^{n}\right), \quad \text { all } \quad T \geq 1,
$$

with $C_{3}=\frac{1}{2} \log 2$.

We will arrange that $2 \mathbb{Z}^{n} \subseteq X \subseteq \mathbb{Z}^{n}$, which guarantees that $X$ is a Meyer set by Corollary 3.1a. We use a simple random construction: pick each point in $\mathbb{Z}^{n} \backslash 2 \mathbb{Z}^{n}$ independently to be in $X$ with probability $1 / 2$. Now consider a box of side $2^{n} T$ centered at a point in $2 \mathbb{Z}^{n}$. Every possible assignment of cells in the box not in the lattice $2 \mathbb{Z}^{n}$ occurs with positive probability, hence for any fixed assignment the possibility that no such configuration occurs in $X$ is zero. The union over all such exceptional events, for $T=1,2,3, \ldots$, then has probability zero, hence with probability one every possible assignment occurs, for every $T \geq 1$. Thus, with probability one,

$$
N_{X}^{*}\left(2^{n} T\right) \geq \exp \left((\log 2)\left(2^{n}-1\right) T^{n}\right), \quad \text { for all } \quad T \geq 1,
$$

as required.

There are much stronger examples known than the one above. A Delone set $X$ of finite type is minimal if it has the repetitivity property: For each $T>0$ there is a finite value $M_{X}(T)$ such that every ball of radius $M_{X}(T)$ contains a copy of each patch of $X$ of radius $T$. Furstenberg [16, Theorem III.2] constructs a symbolic sequence giving a minimal set with positive entropy. By using intervals of different integer lengths to represent the symbols, we obtain a one-dimensional Meyer set which is minimal and satisfies (3.15) for some $C_{3}$. Pleasants [61] constructs such examples in arbitrary dimensions. Minimal sets are important because they are an analogue of "ground state" configurations, see [64], and the introduction to part II. 


\section{Inflation Symmetries}

We recall the basic definition.

Definition 4.1. A Delone set $X$ in $\mathbb{R}^{n}$ has an inflation symmetry by the real number $\eta>1$ if $\eta X \subseteq X$.

If $\mathbf{0} \notin X$, then $X^{\prime}=X \cup\{\boldsymbol{0}\}$ is also a Delone set with an inflation symmetry by $\eta$, so without loss of generality we may assume that $\mathbf{0} \in X$. An inflation symmetry is not a complete symmetry of a Delone set $X$, because the set $X \backslash \eta X$ contains infinitely many points. Inflation similarities occur in Delone sets constructed as control points of self-similar tilings, see [32] and [74].

A (complex) number $\eta$ is an algebraic integer if $f(\eta)=0$ for some nonzero monic polynomial $f(u) \in \mathbb{Z}[u]$, i.e.,

$$
f(u)=u^{n}+\sum_{j=0}^{n-1} a_{j} u^{j} .
$$

The degree of $\eta$ is the minimal degree of any nonzero polynomial $f(u) \in \mathbb{Z}[u]$ with $f(\eta)=0$. The algebraic conjugates of $\eta$ are the other roots of the minimal degree monic polynomial in $\mathbb{Z}[u]$ which $\eta$ satisfies.

Several classes of real algebraic integers appear in studying inflation symmetries.

Definition 4.2. Let $\eta$ be a real algebraic integer with $\eta>1$.

(i) $\eta$ is a Pisot number or Pisot-Vijayaraghavan number if all algebraic conjugates $\left|\eta^{\prime}\right|<1$.

(ii) $\eta$ is a Salem number if all algebraic conjugates $\left|\eta^{\prime}\right| \leq 1$ and at least one $\left|\eta^{\prime}\right|=1$.

(iii) $\eta$ is a Perron number if all algebraic conjugates $\left|\eta^{\prime}\right|<\eta$.

(iv) $\eta$ is a Lind number if all algebraic conjugates $\left|\eta^{\prime}\right| \leq \eta$ and at least one $\left|\eta^{\prime}\right|=\eta$.

Pisot and Salem numbers have been extensively studied, see [4]. Perron numbers were introduced and studied by Lind [46]-[48] and Lind numbers are introduced here.

Theorem 4.1. Let $X$ be a Delone set in $\mathbb{R}^{n}$ such that $\eta X \subseteq X$ for a real number $\eta>1$.

(i) If $X$ is finitely generated, then $\eta$ is an algebraic integer. If $X$ has rank $s$, then the degree of $\eta$ divides $s$.

(ii) If $X$ is a Delone set of finite type, then $\eta$ is an algebraic integer with all algebraic conjugates $\left|\eta^{\prime}\right| \leq \eta$. That is, $\eta$ is a Perron number or a Lind number.

(iii) If $X$ is a Meyer set, then $\eta$ is an algebraic integer with all algebraic conjugates $\left|\eta^{\prime}\right| \leq 1$. That is, $\eta$ is a Pisot number or a Salem number.

Proof. (i) Let $\mathbf{0} \in X$ and suppose that $X$ has $\operatorname{rank} s$, so that

$$
[X]=\mathbb{Z}[X]=\mathbb{Z}\left[\mathbf{v}_{1}, \ldots, \mathbf{v}_{s}\right]
$$


for suitable vectors $\left\{\mathbf{v}_{i}: 1 \leq i \leq s\right\}$ independent over $\mathbb{Q}$. Now $\eta X \subseteq X$ implies that $\eta[X] \subseteq[X]$, hence

$$
\eta \mathbf{V}=\mathbf{M V}, \quad \text { with } \quad \mathbf{V}=\left[\begin{array}{c}
\mathbf{v}_{1} \\
\vdots \\
\mathbf{v}_{s}
\end{array}\right]
$$

in which $\mathbf{M}$ is an $s \times s$ integral matrix and $\mathbf{V}$ is an $s \times n$ real matrix. Now

$$
f(x)=\operatorname{det}(\lambda \mathbf{I}-\mathbf{M}) \in \mathbb{Z}[\lambda]
$$

is a monic polynomial, and $f(\eta)=0$ since $\eta \mathbf{I}-\mathbf{M}$ annihilates an $n$-dimensional subspace of $\mathbb{R}^{s}$. Thus $\eta$ is an algebraic integer, of degree at most $s$.

To show that $d=$ degree $(\eta)$ divides $s$, we use the rational canonical form of $\mathbf{M}$. Let $f(\lambda) \in \mathbb{Z}[\lambda]$ be the minimal degree monic polynomial that $\eta$ satisfies, and write

$$
f(\lambda)=\lambda^{d}+\sum_{j=1}^{d} a_{j} \lambda^{d-j} .
$$

Now (4.2) gives $\eta^{j} \mathbf{V}=\mathbf{M}^{j} \mathbf{V}$ for $1 \leq j \leq d$, hence

$$
f(\mathbf{M}) \mathbf{V}=\left(\mathbf{M}^{d}+\sum_{j=1}^{d} a_{j} \mathbf{M}^{d-j}\right) \mathbf{V}=\mathbf{0} .
$$

Each row of $f(\mathbf{M})$ is an integer vector, so (4.2) gives

$$
\sum_{j=1}^{s} f(\mathbf{M})_{i j} \mathbf{v}_{j}=\mathbf{0}, \quad 1 \leq i \leq s .
$$

The vectors $\mathbf{v}_{j}$ are independent over $\mathbb{Q}$, so it follows that all $f(\mathbf{M})_{i j}=0$, hence $f(\mathbf{M})=$ 0. Thus the minimal polynomial of $\mathbf{M}$ divides $f(\lambda)$, and since $f(\lambda)$ is irreducible over $\mathbb{Q}$, it must equal $f(\lambda)$. It follows that the rational canonical form of $\mathbf{M}$ (over $\mathbb{Q}$ ) can only be a block diagonal matrix with all blocks equal to the $d \times d$ companion matrix $\mathbf{C}_{f}$ of $f$, see p. 199 of [27]. Thus $d$ divides $s$.

(ii) Since $X$ is a Delone set of finite type, by Theorem 2.1 it is finitely generated, of rank $s$, say. Now $\eta$ is a real algebraic integer by (i). Let $\eta$ be of degree $d$ and set $\mathbb{Z}[\eta]:=\mathbb{Z}\left[1, \eta, \ldots \eta^{d-1}\right]$, which is a ring.

We now argue by contradiction, and suppose that $\eta^{\prime}$ is an algebraic conjugate of $\eta$ with $\left|\eta^{\prime}\right|>\eta>1$. We first observe that the $\mathbb{Z}$-module $[X]$ is actually a $\mathbb{Z}[\eta]$-module, because if $\mathbf{y} \in[X]$, then so is $\eta \mathbf{y}$, since we can express $\mathbf{y}$ as a finite integral combination of elements of $X$, and if $\mathbf{x} \in X$, then $\eta \mathbf{x} \in X$. Now [X] is not necessarily a free $\mathbb{Z}[\eta]$ module, but there exists a free $\mathbb{Z}[\eta]$-module $[W]$ in $\mathbb{R}^{n}$ containing $[X]$ as a submodule of finite index. It can be obtained from the matrix converting $\mathbf{M}$ to canonical form in (i) above. Now $d$ divides $s$ and we have

$$
[X] \subseteq[W]=\mathbb{Z}[\eta]\left[\mathbf{w}_{1}, \mathbf{w}_{2}, \ldots, \mathbf{w}_{s / d}\right]
$$


i.e., there is some $\mathbb{Z}$-basis $\left[\mathbf{v}_{1}, \ldots, \mathbf{v}_{s}\right]$ of $[W]$ with

$$
\mathbf{v}_{i+(j-1) s / d}=\eta^{i-1} \mathbf{w}_{j}, \quad 1 \leq i \leq d, \quad 1 \leq j \leq s / d .
$$

We now define the $\mathbb{Z}\left[\eta^{\prime}\right]$-module

$$
\left[W^{\prime}\right]:=\mathbb{Z}\left[\eta^{\prime}\right]\left[\mathbf{w}_{1}, \mathbf{w}_{2}, \ldots, \mathbf{w}_{s / d}\right],
$$

which we regard as a set of points in $\mathbb{C}^{n}$, viewing $\eta^{\prime} \in \mathbb{C}$ even if $\eta^{\prime}$ is real. We call this space $\mathbb{C}^{n}$ the shadow space, and we measure distances on it, using the Hermitian norm

$$
\left\|\mathbf{z}-\mathbf{z}^{\prime}\right\|_{H}^{2}:=\sum_{i=1}^{n}\left|z_{i}-z_{i}^{\prime}\right|^{2}, \quad \text { where } \quad \mathbf{z}=\left(z_{1}, \ldots, z_{n}\right) \in \mathbb{C}^{n} .
$$

We define a shadow map $\sigma:[W] \rightarrow\left[W^{\prime}\right]$ by $\sigma\left(\mathbf{w}_{j}\right)=\mathbf{w}_{j}$ for $1 \leq j \leq s / d$ and extend it using the Galois map from $\mathbb{Z}[\eta]$ to $\mathbb{Z}\left[\eta^{\prime}\right]$, so that

$$
\begin{aligned}
\sigma\left(\mathbf{y}_{1}+\mathbf{y}_{2}\right) & =\sigma\left(\mathbf{y}_{1}\right)+\sigma\left(\mathbf{y}_{2}\right), \\
\sigma\left(\eta \mathbf{y}_{1}\right) & =\eta^{\prime} \sigma\left(\mathbf{y}_{1}\right)
\end{aligned}
$$

for $\mathbf{y}_{1}, \mathbf{y}_{2} \in[X]$. Let $\left[X^{\prime}\right]$ denote the image of $[X]$ under the shadow map, and choose a point $\mathbf{x} \in X$ whose image $\sigma(\mathbf{x}) \neq \mathbf{0}$. We obtain a contradiction by measuring the length of the shadow image of the vectors $\mathbf{y}_{k}=\eta^{k} \mathbf{x}-\mathbf{x}$ in two different ways, as $k \rightarrow \infty$. First, $\sigma\left(\mathbf{y}_{k}\right)=\left(\eta^{\prime}\right)^{k} \sigma(\mathbf{x})-\sigma(\mathbf{x})$ hence

$$
\begin{aligned}
\left\|\sigma\left(\mathbf{y}_{k}\right)\right\| & =\left|\left(\eta^{\prime}\right)^{k}-1\right|\|\sigma(\mathbf{x})\|_{H} \\
& \geq c_{0}\left|\eta^{\prime}\right|^{k} \quad \text { as } \quad k \rightarrow \infty .
\end{aligned}
$$

On the other hand, both $\mathbf{x}, \eta^{k} \mathbf{x} \in X$ and using the Delone property of $X$ we can find a path of elements $\mathbf{x}_{0}, \mathbf{x}_{1}, \ldots, \mathbf{x}_{m} \in X$ from $\mathbf{x}_{0}=\mathbf{x}$ to $\mathbf{x}_{m}=T^{k} \mathbf{x}$, where

$$
\left\|\mathbf{x}_{k}-\mathbf{x}_{k-1}\right\| \leq 3 R, \quad 1 \leq k \leq m,
$$

and $m=(1 / R)\left\|\left(\eta^{k}-1\right) \mathbf{x}\right\|$. Indeed, draw a straight line connecting $\mathbf{x}$ and $\eta^{k} \mathbf{x}$ in $\mathbb{R}^{n}$, and by the Delone property there is a vector $\mathbf{x}_{k} \in X$ within distance $R$ of the point $(1+k(\|\mathbf{X}\| / R)) \mathbf{x}$, and the bound (4.6) follows from the triangle inequality. Since $X$ is of finite type, there are only finitely many vectors in

$$
(X-X) \cap B(\mathbf{0}, 3 R),
$$

hence

$$
C:=\max \left\{\|\sigma(\mathbf{y})\|_{H}: \mathbf{y} \in(X-X) \cap B(0 ; 3 R)\right\}
$$

is finite. It follows from (4.7) that

$$
\left\|\sigma\left(\mathbf{x}_{k}-\mathbf{x}_{k-1}\right)\right\|_{H} \leq C .
$$

Now the linearity of $\sigma$ on $[X]$ gives

$$
\sigma\left(\mathbf{y}_{k}\right)=\sum_{i=1}^{m} \sigma\left(\mathbf{x}_{k}\right)-\sigma\left(\mathbf{x}_{k-1}\right)
$$


hence (4.9) gives

$$
\begin{aligned}
\left\|\sigma\left(\mathbf{y}_{k}\right)\right\|_{H} & \leq \sum_{i=1}^{m}\left\|\sigma\left(\mathbf{x}_{k}\right)-\sigma\left(\mathbf{x}_{k-1}\right)\right\|_{H} \\
& \leq m C=\frac{1}{R}\left(\eta^{k}-1\right)\|\mathbf{x}\| C \\
& \leq C_{1} \eta^{k} \quad \text { as } \quad k \rightarrow \infty
\end{aligned}
$$

If $\left|\eta^{\prime}\right|>\eta$, this contradicts (4.6) for $k$ large enough, so (ii) follows.

(iii) This is a result of Meyer [53, Theorem 6]. Here we present an alternative proof. Since $X$ is a Meyer set, the address map $\varphi:[X] \rightarrow \mathbb{R}^{s}$ is an almost linear map. Let $\tilde{L}: \mathbb{R}^{n} \rightarrow \mathbb{R}^{s}$ be the (unique) linear map such that

$$
|\varphi(\mathbf{x})-\tilde{L}(\mathbf{x})| \leq C, \quad \text { all } \quad \mathbf{x} \in X .
$$

Part (i) of this proof showed that there is an integer matrix $\mathbf{M}$ such that

$$
\varphi(\eta \mathbf{x})=\mathbf{M} \varphi(\mathbf{x}), \quad \text { all } \quad \mathbf{x} \in[X]
$$

and that the minimal polynomial of $\mathbf{M}$ is the monic irreducible polynomial $f(\lambda) \in \mathbb{Z}[\lambda]$ that $\eta$ satisfies. Write

$$
f(\lambda)=\prod_{i=1}^{d}\left(\lambda-\eta_{i}\right)=\lambda^{d}+\sum_{j=1}^{d} a_{j} \lambda^{d-j},
$$

with $\eta=\eta_{1}$. Part (i) also showed that $\mathbf{M}$ is diagonalizable over $\mathbb{C}$, and has a direct sum decomposition into eigenspaces

$$
\mathbb{C}^{n}=V_{1}+V_{2}+\cdots+V_{d}
$$

in which $V_{j}$ is the eigenspace for the eigenvalue $\eta_{j}$. We also have

$$
\mathbf{M v}=\eta_{j} \mathbf{v} \quad \text { for all } \quad \mathbf{v} \in V_{j}, \quad 1 \leq j \leq d,
$$

and $\operatorname{dim}\left(V_{j}\right)=s / d$. The image $\varphi([X])$ spans $\mathbb{R}^{s}$, so there exists $\mathbf{x} \in X$ with $\varphi(\mathbf{x}) \neq \mathbf{0}$. Now $\varphi(\mathbf{x})$ uniquely decomposes into eigenvectors using (4.12), i.e.,

$$
\varphi(\mathbf{x})=\sum_{j=1}^{d} \mathbf{v}_{j}, \quad \text { with each } \quad \mathbf{v}_{j} \in V_{j} .
$$

Since $\varphi(\mathbf{x}) \in \mathbb{Z}^{s} \backslash\{\boldsymbol{0}\}$, every $\mathbf{v}_{j} \neq \mathbf{0}$, because the Galois group of $f(\lambda)$ permutes all the $\mathbf{v}_{j}$ transitively.

Iterating (4.11) gives

$$
\mathbf{M}^{k} \varphi(\mathbf{x})=\sum_{j=1}^{d}\left(\eta_{j}\right)^{k} \mathbf{v}_{j}, \quad \text { all } \quad k \geq 1
$$


On the other hand, (4.10) gives

$$
\left|\tilde{L}\left(\eta^{k} \mathbf{x}\right)-\varphi\left(\eta^{k} \mathbf{x}\right)\right|=\left|\eta^{k} \tilde{L}(\mathbf{x})-\mathbf{M}^{k} \varphi(\mathbf{x})\right| \leq C .
$$

Combining this bound with (4.13) yields

$$
\left\|\eta^{k}\left(L(\mathbf{x})-\mathbf{v}_{1}\right)-\sum_{j=2}^{d}\left(\eta_{j}\right)^{k} \mathbf{v}_{j}\right\| \leq C, \quad \text { all } \quad k \geq 0 .
$$

We claim that this implies that $L(\mathbf{x})=\mathbf{v}_{1}$ and that $\left|\eta_{j}\right| \leq 1$ for all $j \geq 2$. To show this, consider the $i$ th coordinate vector. Then (4.14) gives $\left|h_{k}^{(i)}\right| \leq C$, where

$$
h_{k}^{(i)}=\sum_{j=1}^{d} v_{j i}\left(\eta_{j}\right)^{k}
$$

where $v_{j i}$ is the $i$ th entry of $L(\mathbf{x})-\mathbf{v}_{1}$ for $j=1$ and of $-\mathbf{v}_{j}$ for $j \geq 2$. This relation yields the identity

$$
\sum_{k=0}^{\infty} h_{k}^{(i)} z^{k}=\sum_{j=1}^{d} \frac{v_{j i}}{1-\eta_{j} z}, \quad 1 \leq i \leq s,
$$

where both sides converge as functions of the complex variable $z$ for sufficiently small $|z|$. The relations $\left|h_{k}^{(i)}\right| \leq C$ imply that the power series on the left converges in the unit disk $|z|<1$. Since all $\eta_{j}$ are distinct we must have all $v_{j i}=0$ for any $\left|\eta_{j}\right|>1$. Thus $v_{1 i}=0$ for $1 \leq i \leq s$, hence $L(\mathbf{x})=\mathbf{v}_{1}$. Since each $\mathbf{v}_{j} \neq \mathbf{0}$ for $j \geq 2$, for each such $j$ there exists some $v_{j i} \neq 0$, hence we infer that $\left|\eta_{j}\right| \leq 1$ for $j \geq 2$.

Remarks. Each of (i)-(iii) is apparently sharp, in the sense that given any algebraic integer $\eta$ one can construct a one-dimensional Delone set $X \subseteq \mathbb{R}$ of the appropriate type having $\eta X \subseteq X$. For (iii) this was shown by Meyer. For (i) it is quite easy to give a recursive construction of $X \subseteq \mathbb{R}$, with $[X]=\mathbb{Z}[\eta]$, such that $0 \in X, X=-X$, and $\eta X \subseteq X$. To do this, construct points in $[1, \eta)$, then recursively construct points in $\left[\eta^{k}, \eta^{k+1}\right)$ by first including all points $\eta \mathbf{y}$ for $\mathbf{y} \in\left[\eta^{k-1}, \eta^{k}\right)$, and adding some new points as necessary to preserve the Delone property. For (ii) this construction can apparently be carried out but requires ingenuity in choosing the correct new points to add; I have verified it for $\eta=\sqrt{2}$, which is a Lind number. The proof of Theorem 4.1 above works more generally for real numbers $\eta$ such that $|\eta|>1$, with the condition in (ii) modified to require that all algebraic conjugates $\left|\eta^{\prime}\right| \leq|\eta|$. R. Kenyon (private communication) observes that the Delone set of finite type $X:=\{-n \sqrt{2}: n=1,2, \ldots\} \cup\{n: n=$ $0,1,2, \ldots\}$ has an inflation by $-\sqrt{2}$.

There are results in the literature for self-similar tilings which are analogous to Theorem 4.1. Kenyon [33] proves a result analogous to (i) for tilings. The results for selfsimilar tilings (see [78], [32], and [34]) analogous to cases (ii) and (iii) differ slightly in that they rule out inflation symmetry factors $\eta$ that have an algebraic conjugate $\eta^{\prime}$ which satisfies the equality $\left|\eta^{\prime}\right|=\eta$ in (ii) or $\left|\eta^{\prime}\right|=1$ in (iii). That is, they rule out Lind numbers and Salem numbers in (ii) and (iii), respectively. R. Kenyon (private communication) 
observes that if to Theorem 4.1 one adds the extra hypothesis that the Delone set $X$ is repetitive (i.e., minimal), then Lind numbers can be ruled out in (ii), and Salem numbers can be ruled out in (iii). The repetitivity property holds for self-affine tilings with the unique composition property, see [75].

\section{One-Dimensional Delone Sets of Finite Type and Symbolic Dynamics}

In this section we contrast properties of general Delone sets of finite type and Meyer sets by studying various one-dimensional examples. In particular, we consider diffraction properties of such sets.

One-dimensional Delone sets of finite type $X$ are in one-to-one correspondence with tilings of the line by intervals of finitely many different lengths. If $X=\left\{x_{n}:-\infty<n<\right.$ $\infty$ \} with the points of $X$ numbered in increasing order, then we may regard $X$ as tiling the line with intervals $I_{n}=\left[x_{n}, x_{n+1}\right]$. Theorem 2.1 implies that these intervals have finitely many different lengths, call them $\alpha_{0}, \alpha_{1}, \ldots, \alpha_{m}$. It also implies the converse assertion that any such tiling gives a Delone set $X$ of finite type. Such a tiling is determined by the location of the point $x_{0}$ together with the symbolic expression

$$
S_{X}=\left(\ldots, s_{-1}, s_{0}, s_{1}, \ldots\right) \in\{0,1, \ldots, m\}^{\mathbb{Z}},
$$

in which $s_{i}$ is a symbol indicating the type of the $i$ th interval, and type $j$ denotes an interval of length $\alpha_{j}$. Since we only consider sets $X$ up to translation, we may suppose that $x_{0}=0$, in which case the symbol sequence $S_{X}$ and the assignment of lengths $\alpha_{j}$ to symbol type $j$ completely describe $X$.

We now specialize to the case in which $X$ has intervals of only two lengths, 1 and $\alpha$, in which case a symbol sequence $S \in\{0,1\}^{\mathbb{Z}}$ and the length $\alpha$ completely specify a Delone set $X=X_{\alpha}(S)$ with $x_{0}=0$. We will show that conditions for the sets $X_{\alpha}(S)$ to have a unique autocorrelation measure $\gamma$ and a unique diffraction measure $\hat{\gamma}$ depend on the symbolic address $S$ but not on $\alpha$, while in general the property of $X$ being a Meyer set depends on both $S$ and $\alpha$.

Definition 5.1. A symbol sequence $S \in\{0,1\}^{\mathbb{Z}}$ is almost linear if there is a real number $\beta$ such that the partial sums $S_{n+1}:=s_{0}+s_{1}+\cdots+s_{n}$ and $S_{-n}:=s_{-1}+s_{-2}+\cdots+s_{-n}$, for $n \geq 0$, satisfy

$$
\left|S_{n}-n \beta\right| \leq C, \quad \text { all } \quad n \in \mathbb{Z},
$$

for some constant $C$.

The following result characterizes when $X_{\alpha}(S)$ is a Meyer set.

Theorem 5.1. Let $S \in\{0,1\}^{\mathbb{Z}}$ be a symbol sequence. If $S$ is almost linear, then $X_{\alpha}(S)$ is a Meyer set for all positive $\alpha$. If $S$ is not almost linear, then $X_{\alpha}=X_{\alpha}(S)$ is a Delone set of finite type and satisfies:

(i) If $\alpha$ is rational, then $\operatorname{rank}\left(X_{\alpha}\right)=1$ and $X_{\alpha}$ is a Meyer set.

(ii) If $\alpha$ is irrational, then $\operatorname{rank}\left(X_{\alpha}\right)=2$ and $X_{\alpha}$ is not a Meyer set. 
Proof. If $\alpha \in \mathbb{Q}$, then $X_{\alpha}(S)$ has rank 1 for any $S \subseteq\{0,1\}^{\mathbb{Z}}$. If $\alpha$ has denominator $m$, then $X_{\alpha}(S) \subseteq(1 / m) \mathbb{Z}$, hence $S_{\alpha}(S)$ is a Meyer set by Corollary 3.1a.

If $\alpha \notin \mathbb{Q}$, then if $S \in\{0,1\}^{\mathbb{Z}}$ is not $0^{\infty}$ or $1^{\infty}$, then $X_{\alpha}(S)$ has rank 2 . Now $x_{n}=$ $\left(n-S_{n}\right)+S_{n} \alpha$, so the address map $\varphi:[X] \rightarrow \mathbb{Z}^{2}$ obtained from the basis $v_{1}=1, v_{2}=\alpha$ is

$$
\varphi\left(x_{n}\right)=\left(n-S_{n}, S_{n}\right), \quad \text { all } \quad n \in \mathbb{Z} .
$$

By Theorem $3.1 X_{\alpha}$ is a Meyer set if and only if there exists a linear function $\tilde{L}: \mathbb{R} \rightarrow \mathbb{R}^{2}$, say $\tilde{L}(x)=\left(\beta_{1} x, \beta_{2} x\right)$ such that

$$
\left\|\varphi\left(x_{n}\right)-\tilde{L}\left(x_{n}\right)\right\| \leq C, \quad \text { all } n \in \mathbb{Z},
$$

for some constant $C$. This gives

$\left\|\left(\left(1-\beta_{1}\right) n+\left(\beta_{1} \alpha-\beta_{1}-1\right) S_{n}, \beta_{2} n+\left(\beta_{2} \alpha-\beta_{2}-1\right) S_{n}\right)\right\| \leq C, \quad$ all $\quad n \in \mathbb{Z}$.

Each coordinate is bounded separately, and at least one of them implies (5.2), so that for $X_{\alpha}$ to be a Meyer set, $S$ must be almost linear. Conversely, if $S$ is almost linear, then (5.3) holds for the choice

$$
\beta_{1}=\frac{1-\beta}{1-\beta+\alpha \beta} \quad \text { and } \quad \beta_{2}=\frac{\beta}{1-\beta+\alpha \beta},
$$

so $X_{\alpha}(S)$ is a Meyer set. This includes the cases when $S$ is $0^{\infty}$ and $1^{\infty}$.

Results analogous to Theorem 5.1 hold for sets $X$ derived from symbol sequences drawn from an alphabet with $m$ letters, and tiling $\mathbb{R}$ with intervals of length $\alpha_{0}, \alpha_{1}, \alpha_{2}, \ldots$, $\alpha_{m-1}$.

For results concerning autocorrelation measures and diffraction intensities for onedimensional sets $X$, we rely on results of Hof [24], [26] and Solomyak [73]-[76]. Recall from [24] that an autocorrelation measure $\gamma$ of a Delone set $X$ in $\mathbb{R}^{n}$ is any limit point in the vague topology of the sequence of measures

$$
\gamma^{L}:=(2 L)^{-n} \sum_{\mathbf{x}, \mathbf{x}^{\prime} \in X \cap[-L, L]^{d}} \delta_{\mathbf{x}-\mathbf{x}^{\prime}}, \quad \text { for } \quad L=1,2,3, \ldots
$$

We are primarily interested in sets $X$ that have a unique autocorrelation measure $\gamma$. In order for a unique autocorrelation measure $\gamma$ for $X_{\alpha}(S)$ to exist, the symbol sequence $S$ must have limiting frequencies for the tiles of lengths 1 and $\alpha$, i.e., there must exist a value $\bar{d}$ such that

$$
\lim _{n \rightarrow \infty} \frac{1}{n} S_{n}=\lim _{n \rightarrow \infty} \frac{1}{n} S_{-n}=\bar{d} .
$$

We can easily construct Meyer sets $X$ for which such limits do not exist. For example, take $X=X_{2}(S)$ with $s_{n}=s_{-n}$ and with the $s_{n}$ for $n \geq 0$ being given by

$$
S^{+}=\prod_{k=1}^{\infty}(00)^{2^{k}}(01)^{2^{k}}(11)^{2^{k}} .
$$

Here $X_{2}(S) \subseteq \mathbb{Z}$ is a Delone set with $R=2$, hence is a Meyer set, and (5.4) fails to hold. 
Definition 5.2. (i) A Delone set $X$ of finite type is said to have uniform patch frequencies if for each patch $\mathcal{P}=(X-x) \cap B(0 ; T)$ with $x \in X$, and each $\varepsilon>0$ there is a value $L=L(\mathcal{P}, \varepsilon)$ such that for any interval of length $L$ on the line $X$ contains copies of the patch $\mathcal{P}$ having a density between $d(\mathcal{P})-\varepsilon$ and $d(\mathcal{P})+\varepsilon$. That is,

$$
d(\mathcal{P})-\varepsilon \leq \frac{\#\{y: y+\mathcal{P} \subseteq X \text { and } u \leq y \leq u+L\}}{\#\{x: x \in X \text { and } u \leq x \leq u+L\}} \leq d(\mathcal{P})+\varepsilon .
$$

(ii) We say that a symbol sequence $S \subseteq\{0,1\}^{\mathbb{Z}}$ has uniform patch frequencies if this holds for the Delone set $X_{\alpha}(S)$ obtained from $S$ by taking $\alpha=2$.

It is easy to see that the symbol sequence $S$ has uniform patch frequencies if and only if the same is true for all $X_{\alpha}(S), \alpha>0$. Solomyak [76] observes that this property implies that the tiling dynamical system attached to each such $X_{\alpha}(S)$ is uniquely ergodic, and that each $X_{\alpha}(S)$ has a well-defined autocorrelation measure $\gamma_{\alpha}$ in the sense of Hof [24], [26]. Furthermore, $\gamma_{\alpha}$ is a pure discrete measure supported on $X-X$, with

$$
\gamma_{\alpha}=\sum_{y \in X-X} f(y) \delta_{y}
$$

in which $f(y)$ is the uniform limiting frequency of occurrence of the distance $y$ in $X$. The Fourier transform $\hat{\gamma}_{\alpha}$ of the autocorrelation measure is the diffraction measure of $X_{\alpha}(S)$, and is a positive measure.

Solomyak [74, Theorem 5.1] gives sufficient conditions under which $X$ has uniform limiting patch frequencies. In particular, these conditions are satisfied for $S$ arising as fixed points of irreducible substitutions, and $X=X_{\alpha}(S)$ have the properties above for all $\alpha>0$. Such $X_{\alpha}(S)$ are non-Meyer sets if $\alpha$ is irrational and the largest eigenvalue of the substitution system $S$ is not a Pisot number.

Under the assumption that $S$ has uniform patch frequencies, each set $X_{\alpha}(S)$ has a well-defined diffraction measure $\hat{\gamma}_{\alpha}$. The occurrence of a nonzero discrete component in $\hat{\gamma}_{\alpha}$ depends on both $S$ and $\alpha$, as is shown by examples in [36]. The examples suggest that a two-letter irreducible substitution system can only have a nonzero discrete component when $X_{\alpha}(S)$ is a Meyer set. The associated expansion constant of the substitution $S$ is then necessarily a Pisot number. Solomyak [73] has shown there exist multiletter substitution rules with largest eigenvalue not a Pisot number, which have a discrete component in the spectrum of the associated substitution dynamical system. (See also [13].) At present it is not known whether there exist non-Meyer sets $X_{\alpha}(S)$ with $S$ having uniform limiting patch frequencies, such that the diffraction measure $\hat{\gamma}$ contains some nontrivial discrete spectrum.

Several authors have considered analogous problems for self-similar tilings and tiling dynamical systems. Gähler and Klitzing [18] assert that the set of control points of a self-similar tiling with inflation factor $\eta$ can have a nontrivial discrete component in the diffraction measure only if $\eta$ is a Pisot number. Solomyak in [74, Theorem 8.1] and [75, Theorem 1.4] gives sufficient conditions for a self-similar tiling dynamical system to have a pure discrete spectrum. This result implies that the diffraction measure $\hat{\gamma}$ of any Delone set $X$ constructed from a tiling in the dynamical system by marking tiles is a pure discrete measure. It seems likely that when these conditions are satisfied, the associated Delone set $X$ must be a Meyer set. 


\section{Acknowledgments}

I am indebted to Michael Baake, Ludwig Danzer, Richard Kenyon, Doug Lind, Peter Pleasants, Jim Reeds, Marjorie Senechal, Boris Solomyak, Paul Wright, and the referees for helpful comments. Martin Schlottman supplied a correction concerning Voronoi tesselations on an earlier version of the paper, and suggested including Delaunay tesselations in Theorem 2.2.

\section{References}

1. M. Baake, A guide to mathematical quasicrystals, in: Quasicrystals (J. B. Suck, M. Schrieber, and P. Häussler, Eds.), Springer-Verlag: Berlin, 1999, in press.

2. M. Baake and M. Schlottmann, Geometric aspects of tiling and equivalence concepts, in: Proceedings of the 5th International Conference on Quasicrystals (C. Janot and R. Mossieri, Eds.), World Scientific: Singapore, 1995, pp. 15-21.

3. M. Baake, M. Schlottmann, and P. D. Jarvis, Quasiperiodic tilings with tenfold symmetry and equivalence with respect to local derivability, J. Phys. A 24 (1991), 4637-4654.

4. M. J. Bertin, A. Decomps-Guilloux, M. Grandet-Hugot, M. Pathiaux-Delefosse, and J. P. Schrieber, Pisot and Salem Numbers, Birkhäuser: Boston, 1992.

5. L. Danzer, Quasiperiodicity: local and global aspects, in: Group-Theoretical Methods in Physics (V. V. Dodonov and V. I. Man'ko, Eds.), Lecture Notes in Physics, Vol. 382, Springer-Verlag: New York, 1990, pp. 561-572.

6. L. Danzer, Upper bounds for the lengths of bridges based on Delone sets, Preprint 106, University of Bielefeld, April 1997.

7. L. Danzer and N. Dolbilin, Delone graphs; some species and local rules, in: The Mathematics of Long-Range Aperiodic Order (R. V. Moody, Ed.), Kluwer: Dordrecht, 1997, pp. 98-114.

8. B. Delaunay [B. N. Delone], Sur la sphére vide, Izv. Akad. Nauk SSSR Otdel. Mat. Sov. Nauk 7 (1934), 793-800 (in French).

9. B. N. Delone, A. D. Aleksandrov, and N. N. Padurov, Foundations of the Structural Analysis of Crystals, O.N.T.I.: Leningrad, 1934 (in Russian).

10. D. P. DiVincenzo and P. J. Steinhardt, Eds., Quasicrystals—The State of the Art, World Scientific: Singapore, 1991.

11. N. P. Dolbilin, J. C. Lagarias, and M. Senechal, Multiregular point systems, Discrete Comput. Geom. 20 (1998), 477-498.

12. V. Elser, The diffraction pattern of projected structures, Acta Cryst. Sect. A 42 (1986), 36-43.

13. S. Ferenczi, C. Mauduit, and A. Nogueira, Substitution dynamical systems: algebraic characterization of eigenvalues, Ann. Sci. École Norm. Sup. 29 (1996), 519-533.

14. S. Fortune, Voronoi diagrams and Delaunay triangulations, in: Computing in Euclidean Geometry, 2nd edn. (F. K. Hwang and D. Z. Du, Eds.), World Scientific: Singapore, 1995, pp. 225-265.

15. S. Fortune, Voronoi diagrams and Delaunay triangulations, in: Handbook of Discrete and Computational Geometry (J. E. Goodman and J. O'Rourke, Eds.), CRC Press: Baton Rouge, LA, 1997, pp. 377-388.

16. H. Furstenberg, Disjointness in ergodic theory, minimal sets, and a problem in Diophantine approximation, Math. Systems Theory 1 (1967), 1-49.

17. F. Gähler, M. Baake, and M. Schlottman, Binary tiling quasicrystals and matching rules, Phys. Rev. B 50 (1994), 12458-12467.

18. F. Gähler and R. Klitzing, The diffraction pattern of self-similar tilings, in: The Mathematics of Long-Range Aperiodic Order (R. V. Moody, Ed.), Kluwer: Dordrecht, 1997, pp. 141-174.

19. R. V. Galiulin, Delaunay systems, Soviet Phys. Cryst. 25(5) (1980), 517-520.

20. A. I. Goldman et al., Quasicrystalline materials, Amer. Sci. 84 (1996), 230-241.

21. B. Grünbaum and G. C. Shephard, Tilings and Patterns, Freeman: San Francisco, CA, 1987.

22. C. Henley, Random tilings with quasicrystal order: transfer-matrix approach, J. Phys. A 21 (1988), 16491677. 
23. C. Henley, Random tiling models, in: Quasicrystals: The State of the Art (D. P. DiVincenzo and P. J. Steinhardt, Eds.), World Scientific: Singapore, 1991, pp. 429-524.

24. A. Hof, On diffraction by aperiodic structures, Comm. Math. Phys. 169 (1995), 25-43.

25. A. Hof, On a "Structure Intermediate Between Quasiperiodic and Random," J. Statist. Phys. 84 (1996), 309-320.

26. A. Hof, Diffraction by aperiodic structures, in: The Mathematics of Long-Range Aperiodic Order (R. V. Moody, Ed.), Kluwer: Dordrecht, 1997, pp. 239-268.

27. K. Hoffman and R. Kunze, Linear Algebra, Prentice-Hall: Englewood Cliffs, NJ, 1971.

28. K. Ingersent, Matching rules for quasicrystalline tilings, in: Quasicrystals-The State of the Art (D. P. DiVincenzo and P. J. Steinhardt, Eds.), World Scientific: Singapore, 1991, pp. 185-212.

29. C. Janot, Quasicrystals: A Primer, Oxford University Press: Oxford, 1992.

30. T. Janssen and A. Janner, Incommensurability in crystals, Adv. in Phys. 36 (1987), 519-624.

31. A. Katz, M. Duneau, and C. Oguey, A geometric approach to quasiperiodic tilings, Comm. Math. Phys. 118 (1988), 99-118.

32. R. Kenyon, Self-replicating tilings, in: Symbolic Dynamics and Its Applications (P. Walters, Ed.) American Mathematical Society: Providence, RI, 1992, pp. 239-264.

33. R. Kenyon, Inflationary tilings with similarity structure, Comment. Math. Helv. 69 (1994), 169-198.

34. R. Kenyon, The construction of self-similar tilings, Geom. Funct. Anal. 6 (1996), 471-488.

35. R. Klitzing and M. Baake, Representation of certain self-similar quasiperiodic tilings with perfect matching rules by discrete point sets, J. Phys. France 4 (1994), 893-904.

36. M. Kolàr, B. Iochum, and L. Raymond, Structure factor of ID systems (superlattices) based on two-letter substitution rules: I. Bragg peaks, J. Phys. A 26 (1993), 7343-7366.

37. P. Kramer and R.Neri, On periodic and non-periodic space fillings of $\mathbf{E}^{\mathbf{m}}$ obtained by projection, Acta. Cryst. Sect. A 40 (1984), 580-587. (Erratum, Acta Cryst. Sect. A 41 (1985), 619.)

38. J. C. Lagarias, Meyer's concept of quasicrystal and quasiregular sets, Comm. Math. Phys. 179 (1996), 365-376.

39. J. C. Lagarias, Geometric models for quasicrystals, II. Local rules under isometries, Discrete Comput. Geom., to appear.

40. J. C. Lagarias and P. A. B. Pleasants, Repetitive Delone sets and perfect quasicrystals, preprint.

41. J. C. Lagarias and D. S. Romano, A polyomino tiling problem of Thurston and its configurational entropy, J. Combin. Theory Ser. A 63 (1993), 338-358.

42. T. Q. T. Le, S. A. Piunikhin, and V. A. Sadov, The geometry of quasicrystals, Russian Math. Surveys 48(1) (1993), 37-100.

43. D. Levine and P. J. Steinhardt, Quasicrystals: a new class of ordered structures, Phys. Rev. Lett. 53 (1984), 2477-2480.

44. D. Levine and P. J. Steinhardt, Quasicrystals, I. Definition and structure, Phys. Rev. B 34 (1986), 596-616.

45. L. S. Levitov, Local rules for quasicrystals, Comm. Math. Phys. 118 (1988), 37-100.

46. D. A. Lind, The entropies of topological Markov shifts and a related class of algebraic integers, Ergodic Theory Dynamical Systems 40 (1984), 283-300.

47. D. A. Lind, Matrices of Perron numbers, J. Number Theory 40 (1992), 211-217.

48. D. A. Lind and B. Marcus, An Introduction to Symbolic Dynamics and Coding, Cambridge University Press: Cambridge, 1995.

49. W. F. Lunnon and P. A. B. Pleasants, Quasicrystallographic tilings, J. Math. Pures Appl. 66 (1987), 217-263.

50. N. D. Mermin, Quasicrystallography is better in Fourier space, in: Quasicrystals: The State of the Art (D. P. DiVincenzo and P. J. Steinhardt, Eds.), World Scientific: Singapore, 1991, pp. 133-183.

51. Y. Meyer, Nombres de Pisot, Nombres de Salem, et analyse harmonique, Lecture Notes in Mathematics, No. 117, Springer-Verlag: Berlin, 1970.

52. Y. Meyer, Algebraic Numbers and Harmonic Analysis, North-Holland: Amsterdam, 1972.

53. Y. Meyer, Quasicrystals, Diophantine approximation and algebraic numbers, in: Beyond Quasicrystals (F. Axel and D. Gratis, Eds.), Les Editions de Physique, Springer-Verlag: Paris, 1995, pp. 3-16.

54. R. V. Moody, Meyer sets and the finite generation of quasicrystals, in: Symmetries in Science VIII (P. Gruber, Ed.) Plenum: London, 1995.

55. R. V. Moody, Meyer sets and their duals, in: The Mathematics of Long-Range Aperiodic Order (R. V. Moody, Ed.), Kluwer: Dordrecht, 1997, pp. 403-442.

56. R. V. Moody, Ed., The Mathematics of Long-Range Aperiodic Order Kluwer: Dordrecht, 1997. 
57. R. V. Moody and J. Patera, Colourings of quasicrystals, Canad. J. Phys. 72 (1995), 442-452.

58. K. Mulmuley, Computational Geometry: An Introduction Through Randomized Algorithms, Prentice-Hall: Englewood Cliffs, NJ, 1994.

59. C. Oguey, M. Duneau, and A. Katz, A geometric approach to quasiperiodic tilings, Comm. Math. Phys. 118 (1988), 99-118.

60. S. A. Piunikhin, Quasicrystallographic groups in the sense of Novikov, Mat. Zametki 47(5) (1990), 81-87 [Math. Notes 47 (1990), 478-482].

61. P. A. B. Pleasants, paper in preparation.

62. M. Queffelec, Substitution Dynamical Systems-Spectral Analysis, Lecture Notes in Mathematics, Vol. 1294, Springer-Verlag: New York, 1987.

63. C. Radin, Low temperature and the origin of crystalline symmetry, Internat. J. Mod. Phys. B 1 (1987), 1157-1191.

64. C. Radin, Disordered ground states for classical lattice models, Rev. Math. Phys. 3 (1991), 125-135.

65. C. Radin, Global order from local sources, Bull. Amer. Math. Soc. 25 (1991), 335-364.

66. C. Radin, $\mathbf{Z}^{\mathbf{n}}$ Versus $\mathbf{Z}$ Actions for Systems of Finite Type, Contemporary Mathematics, Vol. 135 American Mathematical Society: Providence, RI, 1992, pp. 339-342.

67. E. A. Robinson, Jr., The dynamical theory of tilings and quasicrystallography, in: Ergodic Theory of $Z^{d}$ Actions (M. Pollicott and K. Schmidt, Eds.), London Mathematical Society Lecture Notes, Vol. 228, Cambridge University Press: Cambridge, 1996, pp. 451-473.

68. C. A. Rogers, Packing and Covering, Cambridge University Press: Cambridge, 1964.

69. M. Schlottmann, Geometrische Eigenschaften quasiperiodischer Strukturen, Dissertation, University of Tubingen, 1993.

70. M. Schlottmann, Cut and project sets in locally compact abelian groups, Proceedings of the Workshop on Aperiodic Order, Fields Institute Communications, American Mathematical Society, Providence, RI, to appear.

71. M. Senechal, Quasicrystals and Geometry, Cambridge University Press: Cambridge, 1995.

72. J. Socolar and P. J. Steinhardt, Quasicrystals, II. Unit cell configurations, Phys. Rev. B 34 (1986), 617-647.

73. B. Solomyak, On the spectral theory of adic transformations, Adv. Soviet Math. 9 (1992), 217-230.

74. B. Solomyak, Dynamics of self-similar tilings, Ergodic Theory Dynamical Systems 17 (1997), 695-738.

75. B. Solomyak, Nonperiodicity implies unique composition for self-similar translationally finite tilings, Discrete Comput. Geom. 20 (1998), 265-279.

76. B. Solomyak, Spectrum of dynamical systems arising from Delone sets, Proceedings of the Fields Institute Conference (J. Patera, Ed.)

77. P. J. Steinhardt and S. Ostlund, Eds., The Physics of Quasicrystals, World Scientific: Singapore, 1987.

78. W. Thurston, Groups, Tilings and Finite State Automata, AMS Colloquium Lecture Notes, Boulder, 1989, American Mathematical Society, Providence, RI, 1989.

Received May 9, 1997, and in revised form March 5, 1998. 